This PDF is a selection from an out-of-print volume from the National Bureau of Economic Research

Volume Title: Annals of Economic and Social Measurement, Volume 5, number 1

Volume Author/Editor: Sanford V. Berg, editor

Volume Publisher: NBER

Volume URL: http://www.nber.org/books/aesm76-1

Publication Date: 1976

Chapter Title: The NBER/NSF Model Comparison Seminar: An Analysis of Results

Chapter Author: Gary Fromm, Lawrence R. Klein

Chapter URL: http://www.nber.org/chapters/c10425

Chapter pages in book: (p. 1 - 28) 


\title{
THE NBER/NSF MODEL COMPARISON SEMINAR: AN ANAI,YSIS OF RESULTS
}

\author{
by Gary Fromm and Lawrence, R. Ki.lin
}

\begin{abstract}
This paper reports comparisons of selected error characteristics and policy multipliers of 11 major econometric models of the U.S. economy. These results were generated by a cooperative effort of the leading model builders under the aegis of the Model Comparison Seminar of the NBER/NSFC Conference on Econometrics and Mathematical Economics. Comparisons of turning point performance. error decomposition. alternative policy analyses. and other characteristics are now underway and will be presented in subsequent seminar symiposia.
\end{abstract}

\section{INTRODUCTION}

For three years, the leading American model builders (macroeconometric) and proprietors have been meeting regularly in a Seminar for the purposes of designing and implementing uniform applications. Basically, the people intimately concerned with model building and maintenance have been dissatisfied with attempts by third party scholars to use the data underlying the models or generated by the models for their own research purposes-often in the form of model testing. Large scale models are such complicated and delicate mechanisins that they require very careful handling by people who fully understand them. While there is some advantage in having the objectivity of third party researchers at work on the problem, there have been so many unfortunate cases of improper use of materials that the Seminar participants have gathered together for their own study of the problem. Through the interaction of group research with group discipline, and the participation of third-party scholars, it is hoped to aclieve the requisite objectivity of comparisons without sacrificing model integrity.

The principal interests of model proprietors in the Seminar work have been focused thus far on error and multiplier analysis. In separate studies, such things as frequency response characteristics, sub-sector performance, specialized policy simulations, optimal control simulations, and other applications have been studied. In the Seminar, we have limited our research to analyses that can readily be made across models, for comparative purposes. ${ }^{1}$

The participating models and proprietors are:

- Bureau of Economic Analysis Model (BEA), A. A. Hirsch, Bruce Grimin, and G. V. L. Narasimham

- Brookings Model. G. Fromm. L. R. Klein and G. Schink

- University of Michigan (MQEM) Model, S. Hymans and H. Shapiro

- Data Resources Inc. (DRI) Model, O. Eckstein, E. Green, and A. Sinai

- Fair Model, Princeton and Yale Universities, R. Fair

'For descriptions of each of the models. see International Economic Review. Vol. 15. No. 2. June 1974. No. 3. October 1974. Vol. 16, No. 1, February 1975. For comparisons of their structures see G. Fromm. "Implications to and from Economie Theory in Models of Complex Systcms." American Journul of Agriculural Economics. May 1973. pp. 259-71. Also see the bibliography at the end of this
article. 
- Federal Reserve Bank of St. L.ouis Model (FRB, St. I .ouis), L. Anderscin and K. Carison

- M.I.I., Pennsylvania, S.S.R.C. Model (MPSS) A. Andu anul R. Rinche

- Wharton Model (Mark $11 I$ and Anticipations Version), M. D. McCarthy, L. R. Klein, F. G. Adams, G. R. Green, and V. Duggal

- Stanford University (H-C Annual) Model. B. Hickman illd R. Cocn

- Wharton Annual Mídicl, R. S. Picsion

- Correll University (Liu-Hwa Monthly) Model, T. C. Lill and H. C. Hwa

A principal feature of the present approach to model comparison and tcsting is the attempt to achieve as much uniformity as scems possible in this area of research. Our collection contains large and small models; annual, quarterly, and monthly models; short and long horizon simulations. It would be both undesirable and unusual if all models were nearly alike.

Still, there are strong similarities among many of the models. With the exception of the monetarist approach of the St. Louis model. all the syste ms follow a Keynesian Framcwork in which expenditurcs depend on incomc and other variables and production or income are functions of expenditures and factor costs. However, there is considerable variation in detailcd specifications and the relative importance accorded financial-real sector interactions in expenditure and portfolio decisions. A limited set of characteristics of the models maty be found in Table 1.

TABIE 1

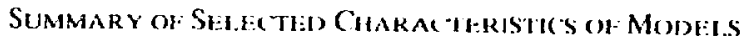

\begin{tabular}{|c|c|c|c|c|}
\hline Model & $\begin{array}{l}\text { Tïne } \\
\text { Frame }\end{array}$ & Scale ${ }^{2}$ & $\begin{array}{l}\text { Disaggregalion } \\
\text { of Production }\end{array}$ & $\begin{array}{c}\text { Endogenous } \\
\text { Financial--Real } \\
\text { Interaction }{ }^{\mathrm{c}}\end{array}$ \\
\hline BEA & Quarterly & Medium & Limited & Weak \\
\hline Brookings & Quarterly & Very Large & Mcdirem & Mediun \\
\hline MQEM & Ouarterly & Small & limited & Weak \\
\hline DRI-74 & Quarterly & Very large & $\begin{array}{l}\text { Medium } \\
\text { (Recursive) }\end{array}$ & Medium \\
\hline Fair & Quarterly & Small & Limited & None \\
\hline St. Louis & Quarterly & Very Small & Nomi & Strong \\
\hline MPS & Quarterly & l.argc & L.imited & Strong \\
\hline Wharton & Quarterly & l.argc & Medium & Mcdium \\
\hline $\begin{array}{l}\text { H-C } \\
\text { Wharton }\end{array}$ & Annual & Medium & l.imitcd & Weak \\
\hline Annual & Annual & Very I arge & High & Medium \\
\hline Liu-Hwa & Monthly & Medium & limited & Mcdiun) \\
\hline
\end{tabular}

\footnotetext{
${ }^{2}$ Based on number of equations: very small $=9$ or less: small $=10-49$; medium $=50-119$ large $=120-199 ;$ very large $=200$ or morc.

${ }^{\mathrm{b}}$ Based on sector detail: limited $=2-5$ sectors; medium $=6-20$ sectors: high $=21$ or more sectors. ${ }^{c}$ Based on qualitative judgnents on jervasiveness of financial variables in real sector cquations
and real variables in financial sector equations.
}

Therefore, the challenging issue is to make comparative sense of standardized applications of differentiated models. 
The directives to model proprictors were to.

I. simulate dynamically from fixed initial conditions

1961:1-1967:4 quarterly

1961:1-1967:12 monthly

$1961-1967$ annually

2. sinulate dynanically from fixed initial conditions beyond sample values

3. calculate fiscal multipliers for changes in non-defense spending or personal income taxes with and without accommodating monctary policies.

In the error calculations for individual variables, we asked for mean-squared error, the variance of error, and the bias computed from the formula,

$$
\begin{aligned}
\text { MSE }=\frac{1}{T} \sum_{t-1}^{T}\left(\hat{X}_{t}-X_{t}\right)^{2} & =\frac{1}{T} \sum_{t}^{T}\left[\left(\hat{X}_{t}-X_{t}\right)-(\overline{\hat{X}}-\bar{X})\right]^{2}+(\overline{\hat{X}}-\bar{X})^{2} \\
\text { MSE } & =\text { VARIANCE }+B I A S^{2} \\
\hat{X}_{t} & =\text { forecast value of } X \\
X_{t} & =\text { observed valuc of } X \\
\overline{\hat{X}} & =\text { mean of } \hat{X}=\frac{1}{T} \sum_{t}^{T} \hat{X}_{t} \\
\bar{X} & =\text { mean of } X=\frac{1}{T} \sum_{t-1}^{T} X
\end{aligned}
$$

In the case of the historical sample period, we standatdized the calculations to the period 1961-67, if possible. In some instances, the samples terminated prior to 1967, and the exercise was accordingly translated or truncated. For the extrapolations beyond the sample period, the starting date for the simulations was right after the end of the sample and therefore not uniform across all models. Data limitations made the spans of the exirapolation period differ for each model. Generally, we looked for 8 period lengths of solution for each simulation exercise with a period being a month, quarter, or year. Some models are not structured to run dynanically that long and others are cut short for diverse reasons. Therefore, all simulations are not of equal length, either for solution span or period covered for the different solutions.

The list of variables simulated is:

GNP, nominal

GNP, real, 1958 prices

GNP, implicit price deflator, 1958: 100

Unemployment rate

Consumer expenditures, nominal

Consumer expenditures, real, 19.58 prices

Nonfarm investment, nominal

Nonfarm investment, real, 1958 prices

Nonfarm inventory investment, nominal

Nonfarm inventory investment, real, 1958 prices 
Residential construction, nominal

Residential construction, real, 1958 prices

Short-term interest rate

Long-term interest rate

Nonfarm wage rate

Hours worked per week

Corporate profits before tax and IVA

Money supply (M1)

Employee compensation, nominal

Personal income, nominal.

The small models (Fair and St. Louis) had no information for several of these variables. In other cases, some variables were obtainable from the models; some were not.

Some of the variables in this list, which is merely an extract from the larger list of variables in several of the models, are conrected through identities. The first three variables satisfy

$$
\frac{\text { GNP (nominai) }}{\text { GNP (real) }}=\text { GNP (deflator) }
$$

All three variables are stochastic, but only two independent pieces of information about stochastic performance can be inferred from the error statistics associated with them. Tabulations are given for all three, but they should not be independently interpreted. Also, profits come from a national income--national product identity in some models; in others there are direct profit equations, and the statistical discrepancy is the "residual." In the profits case as well, in terpretation should be adjusted to the fact that all the components of income may not be independently estimated.

As a study group we set out with high standards for uniformity; but, as in any practical application, we had to allow many compromises. In the end, we achieved about as much uniformity as we could hope to get from 12 teams of independent scholars-especially in economics.

\section{ERROR ANALYSIS}

Before we look into the details of the several models' performance, let us make some overall conclusions on the basis of the error analysis.

1. There are substantial disparities among the different variabies studied for simulation error. Smooth, slow moving variables are more accurately simulated than are variables with high variance and large period-toperiod fluctuation. Among the components of GNP, the largest element by far is consumption, but on an absolute basis, the errors associaled with relatively small magnitudes like fixed investment and inventory investment are as large as the consumption errors. Similarly, on the income side, the errors associated with profits are as large as those associated with wages, although the latter variable is much larger. Also the error in simulating the relatively slow moving long-term interest rate is much 
smaller than the error in simulating the short-term interest rate. Some of the general comments about performance are contradicted for some of the models. These general observations; refer to predominant model performance.

2. Simulation error grows with the length of the simulation period; the error in one-period simulations is smaller than the error in two-period simulations which, in turn, is smaller than the error in throc poriod simulations, etc. 'There are a few exceptions that can be explained by some peculiarities or smallness of sample.

3. There are effectively two regimes-within sample and one in extrapolation. Within sample simulations look very favorable. The error statistics for this group of simulations are about as low as we could expect to realize with "noisy" economic data. If error statistics were actually this small in realistic applications, policymakers would have little to worry about, as far as forecasting precision is concerned. Extrapolation error is. on the other hand, nearly two or three times as large as within-sample simulation error. When one does not have the confines of samples that contain only data to which the model has been "fitted," one is subject to a much wider margin or error. Extrapolation error is just on the borderline of being usable for policy application. There is definitely room for improvement although empirical models with this observed degree of imprecision have proved to be useful in decision-making processes.

4. For central vaı iables like real and nominal GNP, the errors in simulating first differences are smaller than the errors in simulating levels. This is indicative of a significant bias component, which gets "differenced out." In most cases, error accumulation is moderate for simulated first differences.

Table 2 gives results for each model for real and nominal GNP (with first differences, as well), both inside and outside sample periods. The main conclusions (1-4) started above can be seen in this and the succeeding tables. Consider the BEA Model for a start. The GNP error grows from approximately $\$ 2.0$ billion to about $\$ 8.0$ or $\$ 9.0$ billion in 6 quarters; but in first difference form the growth is only from about $\$ 2.0$ to $\$ 4.0$ billion. In some models the first difference errors are essentially flat. Also, the increase of extrapolation error over within-sample error is noticeable in every case. It is hard to characterize this growth, but it would not be an understatement to say that error doubles or triples in extrapolation.

The number of extrapolation periods is extremely limited; therefore, firm statements about extrapolation periods cannot be made. More experience wil! have to be gained with this measure. All models have not becn able to provide extrapolation simulations, and the one, two, or three observations for the longest

\footnotetext{
${ }^{2}$ These remarks should not be confused with those relevant to error of time-cunulated aggregates; thus the error of one-period change in some variables may be less than the error of total change over many periods. where the latter can be calculated as the sum of all intermediate onc-period changes. This kind of cumulation over longer periods of time is used in the paper by leona!l $C$ Andersen and Keith M. Carlson. "St. Louis Model Revisited," International Economic Revie'w. (June. 1964). It was also used in some early error calculations for the Wharton Model. See L. R. Klein and $M$. K. Evans, The Wharton Ouarterly Econometric Forecasting Model 2nd enlarged edition. (Philadelphia: Economic Research Unit, University of Penna., 1968.)
} 
extrapolations were sometimes very close. giving a mislcalding implication of improved forecast accuracy with lengthening horizon in the casc of the Wharton Model (first differences) and the Lill-Hwa Model.

There are nore striking similarities than differences across models. In the short run, GNP prediction errors for one or two quarters (2-6 months for Liu-Hwa) look very much the same, given the error of measurement of GNP itself in most models. Much larger differences show up in extrapolation. although in change form similarity prevails again. The snail models, the Fair Model and the St. Louis Model seem to have rather small GNP errors even in extrapolation, but this may have been a very favorable sample period for them. New economic programs (NEP), shortages, and other rough economic events of later years have been hard on model performance for these two systems. It should be stressed that the Fair model changes every quarter in extrapolation. The up-dating of coeffcients is something like the system of "constant adjustments" made in ex ante forecasting, which serve to keep most of the other models much closer to actual values in ex ante forecasting than would be suggested by the extrapolation crror calculations in Tables $2-5$.

The figures in Table 3 show that consumption errors are of the order of magnitude of GNP errors; they have about the same percentage error as GNP error. Among other leading components of GNP. inventory error is quite large. but it does not grow very much with projection horizon or between within-sample and extrapolation periods. It fluctuates pretty much likc an unexplained random variable with a zero mean. It defies systematic explanation in tight-fitting equations and appears in model simulation to be like a disturbance of the systen as a
whole.

Housing investment and business capital formation have similar error patterns and sizes. They do not grow as much as the consumption error over the simulation horizon, but they have much larger percentage errors than does consumption. In dollar magnitude, the three types of investment (I, II, and IF) contribute more towards total GNP error than does consumption.

In extrapolation, the models got caught up in a highly inflationary environment. The price level error grows considerable with the extrapolation horizon, as does the wage rate projection. The RMSE for the wage rate was remarkably stable over the interpolation simulation horizon. For most models, the short interest rate is subject to larger error than is the long rate. There are only isolated exceptions to
this rule for certain periods in a few models.

On the income side of the national income account figures there is a sinilar classification of stable and volatile items giving rise to a dispersion of error magnitude. Profit error is large relative to the level of profits. The Wharton and BEA Models are exceptional in the extrapolation sinulation.

Money supply is a stock variable and therefore slower-moving than components of GNP or personal income. Errors seem to grow only moderately and in some models do not show such large amplification between extrapolation and within-sample periods. Some models, howeyer, by-pass the endogenous treat-
ment of money supply.

The analytical purpose behind this detailed investigation of model comparison is to look for insights into ways of improving upon model pertormani:- 
Models that are simulated here without any adjustments in extrapolation do worse and residual variables (unemployment, profit) are better projected in systems that build direct estimates of these variables. Actual forecasts wou!d, in fact, make initial corrective adjustments so that errors would be much smaller in such cases than in the unadjusted extrapolations.

It is not intended to try to infer from this cross-model comparison any best model. No mudel iruiy ciominates on the basis of the ground rules laid down here. Some are better on one variable; others on different variables. The differences between models are often so small that they are not significant when errors of measurement are taken into account. An improvement in something like GNP simulation would have to be persistently more than $\$ 1.0$ billion in order to be worth considering, and even that sum is clouded by measurement error. The Wharton anticipations version shows persistently lower errors than does Mark III, but the difference is quite small, at most $\$ 0.5$ billion. This apparent improvement in error performance is suggestive but by no means definitive.

The Liu-Hwa model, after 8 months has about the same GNP error as many of the models after two quariers, over the sample period. In extrapolation, the Liu-Hwa errors are smaller but the saniple is too small to be clearly indicative. One of the brightest hopes for substantial improvement, however, many be in the use of monthly data.

The annual models, after 2 years, show errors that are comparable with quarterly models after 8 quarters. The error of nominal GNP continues to grow after 2 years for the Wharton Annual model, but real GNP errors are quite stable for longer simulations. The I lickman-Coen model simulations are in some cases a bit larger than other model errors for one or two years, but this model's errors stabilize rapidly and do not grow in the third and later years of simulation horizon.

The root mean squared error was decomposed as remarked previously, into a variance and a bias component. The bias component is quite large for some of the main aggregates. That is why the first difference transformation produces markedly smaller errors than for levels of GNP. Other main aggregates such as total consumption or wage payments also have large bias components. Volatile magnitudes such as inventory investment do not have large bias components. In the later, hyperinflationary, period of 1973-74 the tendency to underestimate the price level more than price change is also indicative of a large bias component.

\section{Dynamic Policy Multiplifers}

Examinations of complete-system solution errors within and beyond sample periods over which parameters are estimated, such as those conducted, are useful for indicating how models perform in unconditional prediction. Given actual values of exogenous variables, such tests reveal whether models yield aggregate economic magnitudes sufficiently close to reality so that the results may be used as reliable inputs for subsequent analysis and policy decisions. However, error staístics generally do not reveal much information about responsiveness of models to shifts in policy variables or parameters. That is, they are of limited value for evaluating conditional forecasting. 
TABLE: 2

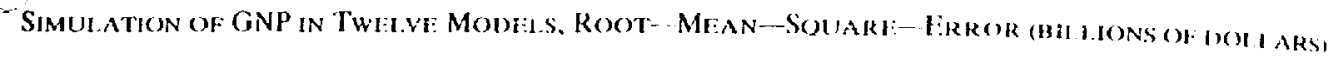

\begin{tabular}{|c|c|c|c|c|c|c|c|c|c|c|c|}
\hline & & & \multicolumn{8}{|c|}{ periods abead - Willin Sample } & \multirow{2}{*}{$\begin{array}{l}\text { long } \\
\text { run }\end{array}$} \\
\hline & & & 1 & 2 & 3 & 4 & 5 & 6 & 7 & $g$ & \\
\hline BEA & real & $\begin{array}{c}\text { GNP\$ } \\
\Delta \\
\text { GNP } \\
\Delta\end{array}$ & $\begin{array}{l}2.34 \\
? 39 \\
1.97 \\
1.97\end{array}$ & $\begin{array}{l}4.68 \\
386 \\
3.94 \\
3.28\end{array}$ & $\begin{array}{l}6.57 \\
419 \\
5.68 \\
3.68\end{array}$ & $\begin{array}{l}781 \\
4.21 \\
6.94 \\
3.78\end{array}$ & $\begin{array}{l}8.95 \\
1.21 \\
8.12 \\
3.76\end{array}$ & $\begin{array}{l}9.94 \\
5.50 \\
8.94 \\
3.48\end{array}$ & & & $\begin{array}{r}1.3 .65 \\
4.45 \\
9.53 \\
4.46\end{array}$ \\
\hline Brookings & real & $\begin{array}{c}\text { GNPS } \\
\Delta \\
\text { GNP } \\
\Delta\end{array}$ & $\begin{array}{l}4.08 \\
4.08 \\
3.70 \\
3.711\end{array}$ & $\begin{array}{l}5.38 \\
3.24 \\
4.66 \\
2.96\end{array}$ & $\begin{array}{l}5.8 .3 \\
3.37 \\
5.01 \\
3.1 .3\end{array}$ & $\begin{array}{l}5.85 \\
3.44 \\
5.1 .3 \\
.3 .26\end{array}$ & $\begin{array}{l}5.78 \\
3.72 \\
5.19 \\
3.43\end{array}$ & $\begin{array}{l}5.72 \\
3.66 \\
5.25 \\
3.38\end{array}$ & $\begin{array}{l}5.616 \\
3.64 \\
5.32 \\
3.37\end{array}$ & $\begin{array}{l}5.811 \\
3.66 \\
5.57 \\
3.3 .3\end{array}$ & \\
\hline MQEM & reat & $\begin{array}{c}\text { GNPS } \\
د \\
\text { GNP } \\
\Delta\end{array}$ & $\begin{array}{l}3.25 \\
3.25 \\
2.97 \\
2.97\end{array}$ & $\begin{array}{l}4.72 \\
5.18 \\
4.8 .3 \\
5.24\end{array}$ & $\begin{array}{l}7.11 \\
7.51 \\
7.11 \\
6.91\end{array}$ & $\begin{array}{l}8.1 .5 \\
6.48 \\
8.27 \\
6.20\end{array}$ & $\begin{array}{l}9.15 \\
6.96 \\
9.35 \\
0.67\end{array}$ & $\begin{array}{r}9.91 \\
7.21 \\
10.14 \\
6.91\end{array}$ & $\begin{array}{r}10.32 \\
6.94 \\
10.55 \\
6.63\end{array}$ & $\begin{array}{r}10.118 \\
6.37 \\
10.35 \\
541\end{array}$ & $\begin{array}{l}6.51 \\
+.29 \\
4.48 \\
4.109\end{array}$ \\
\hline DRI & real & $\begin{array}{c}\text { GNPS } \\
3 \\
\text { GNP } \\
\Delta\end{array}$ & $\begin{array}{l}4.73 \\
4.73 \\
3.47 \\
3.47\end{array}$ & $\begin{array}{l}5.82 \\
4.25 \\
4.91 \\
3.58\end{array}$ & $\begin{array}{l}6.102 \\
4.28 \\
4.78 \\
3.59\end{array}$ & $\begin{array}{l}6.29 \\
4.33 \\
4.60 \\
3.57\end{array}$ & $\begin{array}{l}5.78 \\
4.47 \\
4.74 \\
3.89\end{array}$ & $\begin{array}{l}5.67 \\
4.52 \\
5.58 \\
3.84\end{array}$ & $\begin{array}{l}6.21 \\
4.40 \\
5.96 \\
3.79\end{array}$ & $\begin{array}{l}6.33 \\
4.41 \\
6.23 \\
3.88\end{array}$ & $\begin{array}{l}5.24 \\
4.34 \\
6.30 \\
3.67\end{array}$ \\
\hline Fair & real & $\begin{array}{c}\text { GNPS } \\
\Delta \\
\text { GNP } \\
\Delta\end{array}$ & $\begin{array}{l}2.80 \\
2.80 \\
2.81 \\
2.81\end{array}$ & $\begin{array}{l}4.12 \\
3.13 \\
4.14 \\
3.81\end{array}$ & $\begin{array}{l}4.49 \\
3.47 \\
4.32 \\
3.15\end{array}$ & $\begin{array}{l}4.50 \\
3.50 \\
4.22 \\
3.33\end{array}$ & $\begin{array}{l}4.00 \\
3.76 \\
3.61 \\
3.51\end{array}$ & & & & \\
\hline
\end{tabular}

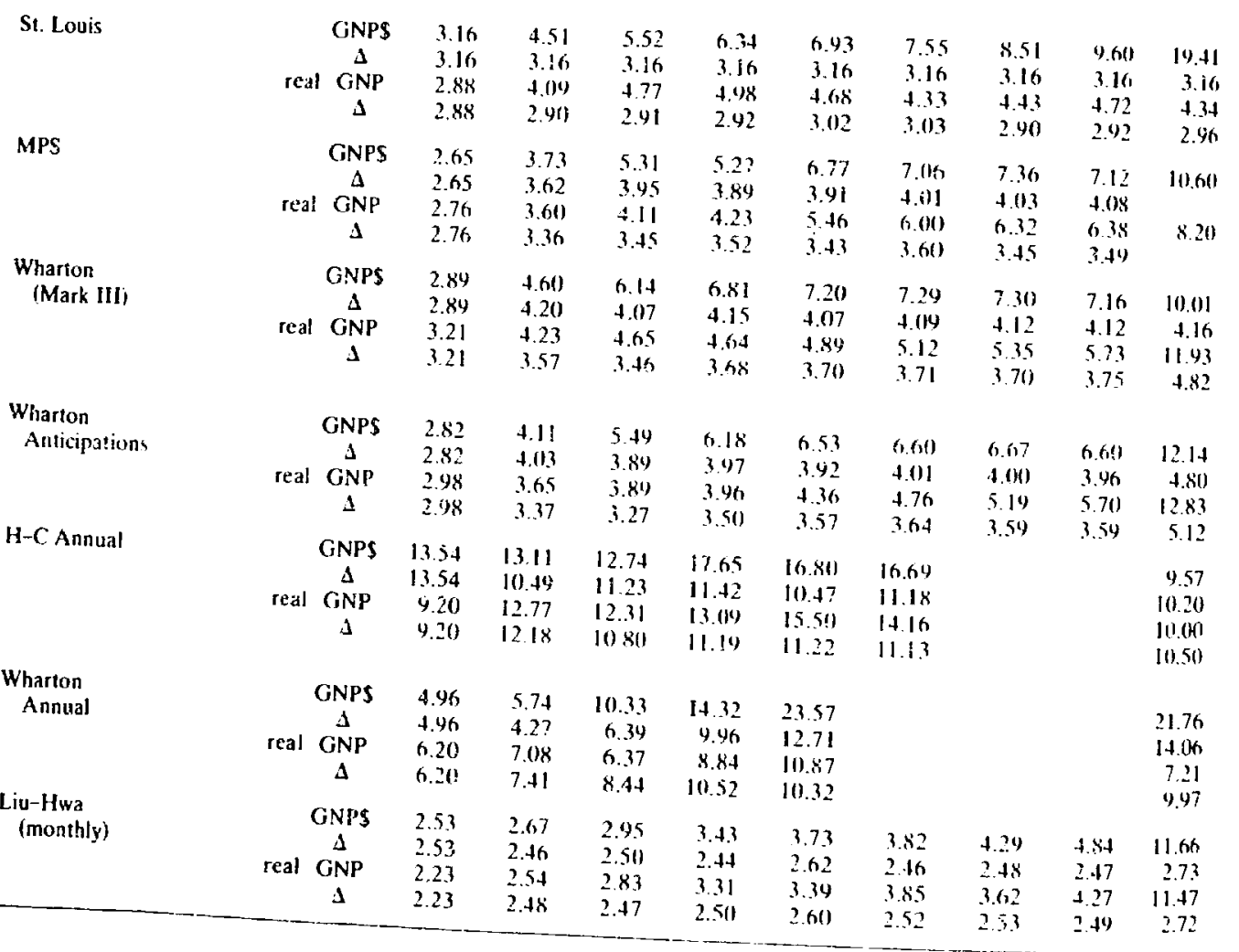




\begin{tabular}{|c|c|c|c|c|c|c|c|c|}
\hline \multicolumn{8}{|c|}{ periods ahead-F:xlrapulation } & \multirow[b]{2}{*}{ Notes } \\
\hline 1 & 2 & 3 & 4 & 5 & 6 & 7 & 8 & \\
\hline $\begin{array}{l}4.30 \\
4.30 \\
3.51 \\
3.51\end{array}$ & $\begin{array}{r}12.47 \\
11.31 \\
9.0 .5 \\
7.93\end{array}$ & $\begin{array}{r}18.21 \\
6.40 \\
1 i .54 \\
3.38\end{array}$ & $\begin{array}{r}20.78 \\
4.04 \\
\text { i i.62 } \\
3.48\end{array}$ & $\begin{array}{r}21.13 \\
4.09 \\
8.42 \\
5.56\end{array}$ & $\begin{array}{r}19.72 \\
4.43 \\
0.8 .3 \\
6.81\end{array}$ & & & $\begin{array}{l}\text { Serial correlations of residuals are used } \\
\text { in simuliation as estimated in the sample: } \\
\text { noother adjustments except for the Gin1 } \\
\text { strike. } 1964 \text {. lixtrapolation period is } \\
\text { t969: } 1-1971: 2 \text {. }\end{array}$ \\
\hline $\begin{array}{l}6.74 \\
6.74 \\
5.86 \\
5.86\end{array}$ & $\begin{array}{r}11.36 \\
7.61 \\
9.64 \\
6.311\end{array}$ & $\begin{array}{r}16.08 \\
8.32 \\
13.40 \\
6.90\end{array}$ & $\begin{array}{r}20.94 \\
7.94 \\
16.41 \\
6.47\end{array}$ & $\begin{array}{r}2.5 .64 \\
8.44 \\
18.78 \\
6.84\end{array}$ & $\begin{array}{r}29.54 \\
7.11 \\
20.45 \\
5.75\end{array}$ & $\begin{array}{r}33.18 \\
7.08 \\
21.24 \\
5.81\end{array}$ & $\begin{array}{r}34.77 \\
8.01 \\
24.22 \\
6.34\end{array}$ & $\begin{array}{l}\text { No adjustments made to model as estimated } \\
\text { for within sample simulation. Period is } \\
1959: 1-1965: 4 \text {. Extrapolation period is } \\
1966: 1-1970: 4 \text {. Fatrapolation solution } \\
\text { adjusted for average error in last } 4 \text { sample } \\
\text { periods. }\end{array}$ \\
\hline $\begin{array}{l}6.04 \\
6.04 \\
5.16 \\
5.16\end{array}$ & $\begin{array}{l}9.88 \\
8.07 \\
8.38 \\
6.78\end{array}$ & $\begin{array}{r}12.45 \\
8.60 \\
9.95 \\
7.45\end{array}$ & $\begin{array}{r}16.49 \\
9.35 \\
12.09 \\
7.92\end{array}$ & & & & & $\begin{array}{l}\text { No adjuetments mide lo nodel as estinated } \\
\text { for within sample simulation. Extrapolation } \\
\text { perios is } 1968: 1-1970: 4 \text {. Fxtrapolation } \\
\text { solution adjusted for average treor at end of } \\
\text { sample period. } \\
\text { Model re-estimated in 1974. Within sample } \\
\text { simulation. } 1962: 1-1968: 4 \text {. Extr apolation } \\
\text { not possible with this new version. }\end{array}$ \\
\hline $\begin{array}{l}2.91 \\
2.91 \\
3.12 \\
3.11\end{array}$ & $\begin{array}{l}4.35 \\
3.76 \\
4.74 \\
3.15\end{array}$ & $\begin{array}{l}4.52 \\
4.32 \\
4.71 \\
3.23\end{array}$ & $\begin{array}{l}6.77 \\
4.50 \\
5.40 \\
3.0 .3\end{array}$ & $\begin{array}{l}9.84 \\
4.49 \\
6.61 \\
2.98\end{array}$ & & & & $\begin{array}{l}\text { No adjustment made to motel as estimated } \\
\text { within sample period, } 1962: 1-1967: 4 . \\
\text { hut observed values of anticipation } \\
\text { variabies used as exogenous input. Strike } \\
\text { (y+arters (1964:4. 1965:1.1965:2) deleled. } \\
\text { Coefficients re-estimated every period for } \\
\text { extrapolation. 1965:4-1969:4. }\end{array}$ \\
\hline $\begin{array}{r}10.29 \\
10.29 \\
6.81 \\
6.81\end{array}$ & $\begin{array}{r}14.88 \\
10.89 \\
854 \\
7.04\end{array}$ & $\begin{array}{r}13.83 \\
11.56 \\
8.36 \\
7.62\end{array}$ & $\begin{array}{r}11.69 \\
12.62 \\
10.25 \\
8.18\end{array}$ & $\begin{array}{r}11.15 \\
13.13 \\
8.33 \\
7.77\end{array}$ & $\begin{array}{r}16.11 \\
10.75 \\
10.86 \\
5.33\end{array}$ & & & $\begin{array}{l}\text { No adjustments nuade to model as estimated } \\
\text { for within or outside sample sinulations. } \\
\text { Extrapolation period is } 1970: 1-1971: 4 \text {. } \\
\text { Serial correlations of residuals arc nsed in } \\
\text { simulation as estimated in the sinnple. no } \\
\text { other adjustments. }\end{array}$ \\
\hline $\begin{array}{l}5.71 \\
5.71 \\
5.02 \\
5.02\end{array}$ & $\begin{array}{r}17.04 \\
14.05 \\
12.9 .3 \\
9.67\end{array}$ & $\begin{array}{r}25.09 \\
10.41 \\
17.96 \\
6.71\end{array}$ & $\begin{array}{r}27.25 \\
7.40 \\
19.35 \\
5.00\end{array}$ & $\begin{array}{r}34.14 \\
8.26 \\
21.24 \\
5.14\end{array}$ & $\begin{array}{r}40.35 \\
6.90 \\
21.55 \\
3.72\end{array}$ & $\begin{array}{r}43.99 \\
4.43 \\
19.7 .3 \\
3.57\end{array}$ & $\begin{array}{r}46.57 \\
4.95 \\
17.03 \\
3.45\end{array}$ & 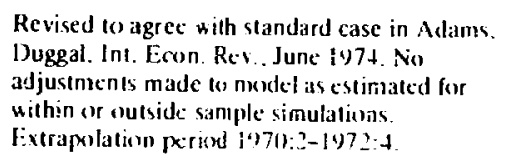 \\
\hline $\begin{array}{l}7.07 \\
7.07 \\
5.180 \\
5.80\end{array}$ & $\begin{array}{r}17.66 \\
12.39 \\
13 .(40 \\
8.80\end{array}$ & $\begin{array}{r}23.16 \\
8.34 \\
16.14 \\
5.49\end{array}$ & $\begin{array}{r}23.40 \\
6.58 \\
16.07 \\
5.08\end{array}$ & $\begin{array}{r}28.60 \\
7.14 \\
16.56 \\
4.87\end{array}$ & $\begin{array}{r}34.02 \\
6.35 \\
16.21 \\
3.56\end{array}$ & $\begin{array}{r}36.79 \\
4.01 \\
14.01 \\
3.74\end{array}$ & $\begin{array}{r}38.01 \\
4.013 \\
10.65 \\
3.66\end{array}$ & 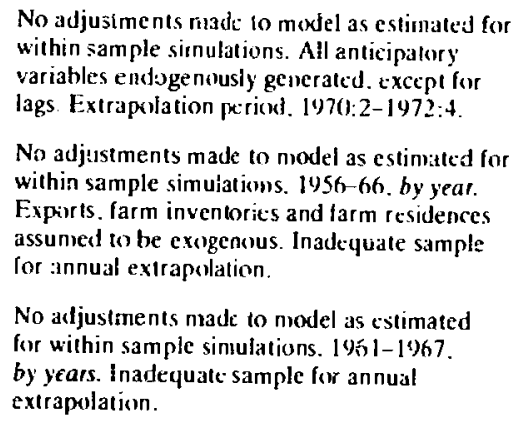 \\
\hline $\begin{array}{l}5.94 \\
5.94 \\
5.29 \\
5.29\end{array}$ & $\begin{array}{l}5.44 \\
5.80 \\
6.19 \\
5.53\end{array}$ & $\begin{array}{l}5.92 \\
6.88 \\
6.19 \\
5.34\end{array}$ & $\begin{array}{l}6.28 \\
5.87 \\
7.88 \\
5.91\end{array}$ & $\begin{array}{l}6.50 \\
5.65 \\
7.76 \\
6.15\end{array}$ & $\begin{array}{l}5.09 \\
5.75 \\
6.66 \\
5.22\end{array}$ & $\begin{array}{l}3.28 \\
5.58 \\
7.67 \\
5.76\end{array}$ & $\begin{array}{l}4.24 \\
5.6 .3 \\
7.38 \\
5.74\end{array}$ & $\begin{array}{l}\text { No adjustments made to model as cstimated for } \\
\text { within or outside sample simulatiuns. sample. } \\
1961:\{1-1967: 12 \text {. outside sample } \\
1972: 011-1972: 12 \text {. }\end{array}$ \\
\hline
\end{tabular}


TABI.I: 3

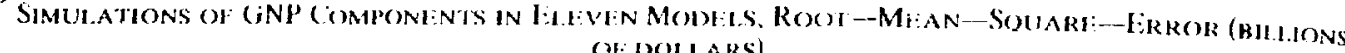
OF BOIIARS

\begin{tabular}{|c|c|c|c|c|c|c|c|c|c|c|}
\hline & & & \multicolumn{8}{|c|}{ periekl ahead - - within sample } \\
\hline & & & 1 & 2 & 3 & 4 & 5 & 0 & 7 & $x$ \\
\hline \multirow[t]{8}{*}{ BEA } & & Cs & 1.91 & 2.58 & 3.08 & 3.76 & 4.59 & 524 & & \\
\hline & réal & $C$ & 1.80 & 2.47 & 3.118 & 3.84 & 4.54 & 4.98 & & \\
\hline & & is & 1.00 & 1.84 & 247 & 3.00 & 3.6 .3 & 4.08 & & \\
\hline & rťal & 1 & 11.96 & $1.6 ?$ & 2.17 & 2.68 & 3.12 & 3.47 & & \\
\hline & & 115 & 2.25 & 3.115 & 3.32 & 3.43 & 3.50 & 3.70 & & \\
\hline & real & II & 2.12 & 2.86 & 3.10 & 3.19 & 3.33 & 3.5 .3 & & \\
\hline & & IH $\$$ & 0.57 & 0.77 & 0.97 & 0.96 & 0.94 & $1.0 !$ & & \\
\hline & real & $1 \mathrm{H}$ & 0.52 & 0.64 & (1).S1 & 0.82 & (I.S? & 0.87 & & \\
\hline \multirow[t]{8}{*}{ Brookings } & & CS & 2.44 & 2.77 & 2.47 & 2.91 & 2.88 & 2.88 & 2.70 & 2.74 \\
\hline & ro:al & $c$ & 2.20 & 2.58 & 2.711 & 2.6 .3 & 2.61 & $2.6 ?$ & 2.59 & 2.72 \\
\hline & & is & 0.59 & 10.84 & 1.(1) & 1.16 & $1.11)$ & 1.22 & 1.27 & 1.43 \\
\hline & real & 1 & 0.52 & $(1.78$ & 0.9 .3 & 1.05 & 1.11 & 1.16 & 1.22 & 1.37 \\
\hline & & 115 & 177 & 2.26 & 2.20 & 2.301 & 2.41 & 2.45 & 2.53 & 2.59 \\
\hline & real & 11 & 1.75 & 2.23 & 2.26 & 2.28 & 2.39 & 2.43 & 2.51 & 2.57 \\
\hline & & IH\$ & 0.5 .3 & 0.90 & 0.95 & 0.93 & 0.41 & 0.96 & $1 .(9)$ & $1.0 !$ \\
\hline & real & 111 & 0.44 & 0.75 & 0.77 & 0.74 & 0.7 .5 & 0.80 & 0.84 & 0.84 \\
\hline \multirow[t]{8}{*}{ MQEM } & & CS & 1.97 & 3.05 & 4.17 & 5.13 & 5.80 & 6.30 & 6.67 & 6.80 \\
\hline & real & $\mathrm{C}$ & 2.15 & 3.20 & 4.41 & 5.45 & 6.08 & 6.52 & $6 . \%)$ & 6.88 \\
\hline & & is & 1.0 .3 & 1.73 & 2.34 & 2.82 & 3.22 & $3+5$ & 3.56 & 3.37 \\
\hline & rest & $!$ & 0.94 & 1.52 & 2.04 & 2.42 & 2.711 & 2.84 & 2.85 & 2.68 \\
\hline & & 115 & & & & & & & & \\
\hline & real & II & 2.3 .3 & 2.45 & 2.57 & $\begin{array}{r}2.47 \\
135\end{array}$ & 2.70 & 2.93 & 2.88 & 2.90 \\
\hline & & IHS & 0.67 & 0.91 & 1.22 & 1.25 & 1.27 & 1.30 & 1.54 & 1.60 \\
\hline & real & IH & 0.54 & 0.76 & 1.01 & 1.05 & 1.04 & 1.17 & I. 28 & 1.32 \\
\hline \multirow[t]{8}{*}{ DRI } & & $\mathrm{Cs}$ & 3.35 & 3.82 & 3.86 & 4.17 & 4.16 & $4 .(+9)$ & 4.06 & 3.89 \\
\hline & real & c & 3.06 & 3.42 & $3.28^{2}$ & 3.10 & 3.211 & 3.48 & 3.65 & 3.69 \\
\hline & & IS & 1.62 & 1.81 & 2.15 & 2.20 & 2.28 & 2.41 & 2.51 & 2.65 \\
\hline & real & 1 & 140 & 1.53 & 1.8 .5 & 1.94 & 2.03 & 2.17 & 2.29 & 2.41 \\
\hline & & IIs & 2.08 & 2.42 & 2.48 & 2.56 & 2.59 & 2.6 .3 & 2.74 & 2.87 \\
\hline & real & II & 1.91 & 2.21 & 2.27 & 2.35 & 2.38 & 2.44 & 2.55 & 2.67 \\
\hline & & 1115 & 0.68 & i. (1) & 1.38 & 1.6 .5 & $1.8 k$ & 2.19 & 2.37 & 2.33 \\
\hline & real & IH & 0.57 & $0.81\}$ & 1.11 & 1.35 & 1.50 & 1.87 & 2.100 & 1.93 \\
\hline \multirow[t]{4}{*}{ Fair } & & CS & 1.96 & 2.31 & 2.79 & 298 & 3.17 & & & \\
\hline & real & 1 & & & & & 1.11 & & & \\
\hline & & $\begin{array}{l}\text { I1\$ } \\
\text { II }\end{array}$ & 2.56 & 3.43 & 3.35 & 3.42 & 2.46 & & & \\
\hline & & & & & & & & & & \\
\hline \multirow[b]{9}{*}{ Nharion Mark IIl } & real & c & $\frac{2.12}{2.01}$ & 2.81 & .3 .24 & 3.36 & 3.41 & 4.42 & 4.82 & 4.79 \\
\hline & & is & $\begin{array}{l}2.01 \\
1109\end{array}$ & 2.90 & 3.80 & 3.88 & 4.66 & 5.15 & 5.50 & 5.601 \\
\hline & real & 1 & 1.99 & 1.21 & 1.34 & 1.47 & 1.59 & 1.68 & 1.74 & 1.79 \\
\hline & & UIS & 1.02 & 1.28 & 1.51 & 1.68 & 188 & 1.98 & 201 & 1.49 \\
\hline & real & [I & 2.01 & 2.09 & 2.14 & 2.19 & 2.49 & $2.4 k$ & 2.56 & 255 \\
\hline & & IHs & 2.11 & 2.19 & 2.25 & 2.32 & 2.6 .3 & 2.62 & 2.72 & 2.70 \\
\hline & & 113 & 0.43 & $0.6 ?$ & 0.8 .3 & 0.80 & 11.81 & 11.91 & 108 & 1.22 \\
\hline & real & If & 0.19 & 0.71 & 11.94 & 0.89 & $0 . \% 0$ & l. (h) & 1.21 & i. 38 \\
\hline & & Cs & 1.92 & 2.53 & $3.3(1)$ & $3 . \times 7$ & 4.2 .3 & +.52 & 4.66 & +.80 \\
\hline \multirow{7}{*}{ Wharion Mark III } & real & $c$ & 2.010 & 2.46 & 2.76 & 2.88 & $3 .(60)$ & 3.16 & 3.25 & 3.22 \\
\hline & & IS & 1.95 & 2.23 & 2.42 & 2.47 & 2.51 & 2.52 & 2.53 & 2.52 \\
\hline & real & 1 & 1.82 & 1.97 & 2.05 & 2.08 & 2.14 & 2.22 & 2.30 & 2.37 \\
\hline & & 115 & 3.45 & 3.64 & 3.74 & 3.68 & 3.72 & 3.72 & 3.78 & 3.86 \\
\hline & real & II & 3.32 & 3.50 & 3.78 & 3.51 & 3.53 & 3.53 & 3.57 & 3.65 \\
\hline & & $1 \mathrm{HS}$ & 1.84 & $1 \times 9$ & 2.192 & 2.14 & 2.17 & 2.21 & 2.24 & 2.27 \\
\hline & real & IH & 1.57 & 1.57 & 1.67 & 1.70 & 1.87 & 1.45 & 2.01 & 2.03 \\
\hline
\end{tabular}




\begin{tabular}{|c|c|c|c|c|c|c|c|c|}
\hline \multirow{2}{*}{$\begin{array}{l}\text { long } \\
\text { run }\end{array}$} & \multicolumn{8}{|c|}{ periods ahead-extrapolation } \\
\hline & 1 & 2 & 3 & 4 & 5 & h & 7 & $x$ \\
\hline 9.115 & 4.61 & 4.12 & 13.41 & 16.20 & 18.46 & $20.0 \mathrm{~s}$ & & \\
\hline $5.9 !$ & 4.26 & 7.21 & 9.41 & 10.02 & 9.81 & 9.16 & & \\
\hline 5.55 & 2.01 & 2.32 & 2.59 & 4.112 & 4.8 .5 & 4.57 & & \\
\hline 4.10 & 1.6 .1 & 1.92 & 1.84 & 2.5 .3 & 2.81 & 2.28 & & \\
\hline 3.70 & 2.59 & 5.41 & 6.95 & 6.45 & 4.60 & 2.22 & & \\
\hline 3.44 & 2.18 & 4.50 & 5.84 & 5.42 & 3.86 & $1.9 \%$ & & \\
\hline 1.59 & 1.74 & 3.82 & 4.44 & 4.09 & 3.55 & $3.1 k$ & & \\
\hline \multirow[t]{9}{*}{1.63} & 1.14 & 2.49 & $2 \times 5$ & 2.51 & 2.08 & 1.66 & & \\
\hline & 5.80 & 8.45 & 11.56 & 13.62 & 15.50 & 16.65 & 17.24 & 18.56 \\
\hline & 516 & 7.19 & 9.56 & 10.98 & 11.73 & 12.35 & 11.61 & 12.14 \\
\hline & 3.12 & 3.79 & 4.44 & 5.37 & $f, t, 6$ & 8.14 & 9.72 & 11.31 \\
\hline & 2.57 & 3.37 & 4.14 & 5.03 & 5.47 & 6.85 & 7.58 & 7.9 .4 \\
\hline & 3.08 & $4.6 x$ & 5.01 & 5.47 & 5.115 & 5013 & 4.53 & 423 \\
\hline & 3.37 & 4.27 & +49 & $4.9(1)$ & 4.48 & 4.46 & 3.94 & 3.66 \\
\hline & 1.06 & 2.38 & 3.20 & 3.76 & 3.20 & 3.60 & 3.91 & 463 \\
\hline & 0.80 & 1.83 & 2.48 & 2.93 & 2.97 & 2.82 & 2.93 & 3.30 \\
\hline 4.22 & 4.46 & 6.87 & 8.18 & 10.06 & & & & \\
\hline 7.00 & 4.07 & 6.54 & 7.60 & $8.3 ?$ & & & & \\
\hline 2.94 & 2.42 & 3.54 & .3 .92 & 5.24 & & & & \\
\hline 2.31 & 2.09 & 2.12 & 3.11 & +.30 & & & & \\
\hline 2.39 & 2.75 & 2.52 & 2.78 & 2.97 & & & & \\
\hline 1.53 & 0.85 & 1.23 & 1.46 & 1.40 & & & & \\
\hline 1.34 & 0.67 & 0.97 & 1.16 & 1.02 & & & & \\
\hline \multirow{10}{*}{$\begin{array}{l}3.51 \\
3.81 \\
2.83 \\
2.60 \\
2.83 \\
2.63 \\
2.29 \\
1.92\end{array}$} & & & & & & & & 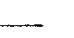 \\
\hline & \multirow[t]{3}{*}{ Notation: } & \multirow{3}{*}{\multicolumn{6}{|c|}{$\begin{aligned} C & =\text { consumer expenditure } \\
1 & =\text { nonfarm gross investment in plant and cquipment } \\
11 & =\text { nonfarm inventory insestment } \\
1 H & =\text { residential construction }\end{aligned}$}} & \\
\hline & & & & & & & & \\
\hline & & & & & & & & \\
\hline & & & & & & & & \\
\hline & & & & & & & & \\
\hline & 3.27 & 3.97 & 4.59 & 6.15 & 7.97 & & & \\
\hline & 1.63 & 1.84 & 2.115 & 2.54 & 3.01 & & & \\
\hline & 3.81 & 4.77 & 4.92 & 4.63 & 4.84 & & & \\
\hline & 1.06 & 2.03 & $3.6)$ & 3.67 & 4.44 & & & \\
\hline 7.12 & & & & & & & & \\
\hline 4.97 & & & & & & & & \\
\hline 2.68 & & & & & & & & \\
\hline 3.21 & & & & & & & & \\
\hline 3.34 & & & & & & & & \\
\hline 3.16 & & & & & & & & \\
\hline 160 & & & & & & & & \\
\hline 1.53 & & & & & & & & \\
\hline 4.97 & 5.87 & 10.43 & 14.82 & 17.33 & 21.64 & 25.40 & 28.32 & 30.36 \\
\hline 6.51 & 2.89 & 5.04 & 7.21 & 8.51 & 9.31 & 9.25 & 8.53 & 6.97 \\
\hline 3.52 & 4.46 & 3.41 & 2.18 & 1.3 .5 & 3.50 & 6.21 & 8.13 & 8.8 .3 \\
\hline 3.26 & 2.03 & 1.65 & 2.13 & 2.59 & 3.58 & 4.79 & 5.30 & 5.17 \\
\hline 5.08 & 4.53 & 8.85 & 12.15 & 12.70 & 14.20 & 14.85 & 14.55 & 14.76 \\
\hline 4.73 & 3.74 & 7.38 & 10.08 & 10.52 & 11.62 & 12.01 & 11.57 & 11.50 \\
\hline 2.40 & 4.02 & 3.62 & 5.32 & 7.91 & 8.80 & 9.17 & 9.51 & 9.68 \\
\hline 2.13 & 3.27 & 3.29 & 4.39 & 6.00 & 6.59 & 6.88 & 7.18 & 7.39 \\
\hline
\end{tabular}




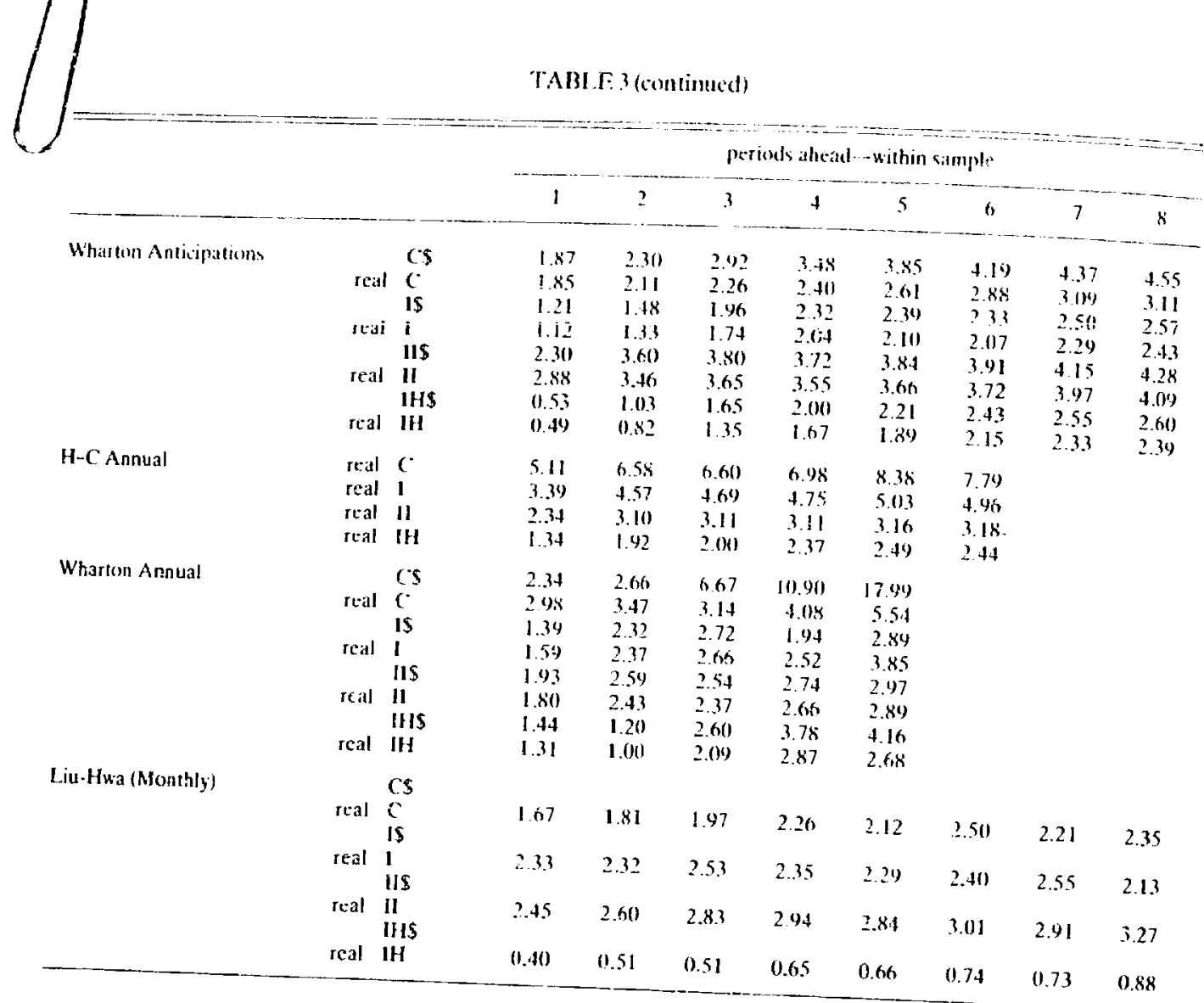

For this reason there is keen interest in dynamic multipliers resulting from alternative monetary and fiscal policy actions. This is particularly.true during the past few years- when there have been massive shifts in government expenditures, taxes, and monetary policy due to the Vietnamese war and the battle to contain inflation. There has been much debate about desired spending and monetary expansion rates. Some differences in prescriptions have come from differences in goals; others have arisen due to controversy about magnitudes of multipliers. number of factors, the relative importance of which is yet to be determined. They are listed here in no particular order. First, lack of standardization of variables treated exogenously probably is a major contributor to discrepancies. For example, a model that has an exogenous foreign sector normally will have, other things being equal, higher GNP-foreign sector government expenditure multipliers than a model that makes imports and exports functions of domestic and foreign incomes and prices. Similarly, expenditure multipliers are downward biased when state and local government outlays are taken to be exogenous. Many other such examples could be given, including those from the financial-monetary sector.

Another cause for discrepancies are differences in periods over which capacity utilization or unemployment rates) and prices, increments in nominal or 


\begin{tabular}{|c|c|c|c|c|c|c|c|c|}
\hline \multirow{2}{*}{$\begin{array}{l}\text { Inno } \\
\text { run }\end{array}$} & \multicolumn{8}{|c|}{ periods ahead-extrapolation } \\
\hline & 1 & 2 & 3 & 4 & 5 & 6 & 7 & 8 \\
\hline 5.59 & 5.38 & 9.33 & 13.16 & 15.1\% & 18.64 & 21.66 & 23.68 & 24.68 \\
\hline 6.46 & 2.46 & 4.16 & 5.87 & 6.71 & 6.75 & 6.114 & 4.72 & 2.55 \\
\hline 4.52 & 1.93 & 2.11 & 2.68 & 3.25 & 2.63 & 3.62 & 6.01 & 6.36 \\
\hline 3.88 & 2.29 & 2.80 & 200 & 102 & 0.06 & 2.54 & 3.73 & 3.49 \\
\hline 6.50 & 6.20 & 6.01 & 9.82 & 10.57 & 12.26 & 11.93 & 10.65 & 10.31 \\
\hline 6.07 & 5.09 & 5.05 & 8.18 & 8.80 & 10.09 & 9.74 & 8.56 & 8.08 \\
\hline 3.57 & 2.72 & 1.12 & 2.85 & 4.57 & 5.98 & 6.50 & 7.07 & 7.25 \\
\hline 3.14 & 1.80 & 1.66 & 2.87 & 3.94 & 4.89 & 5.30 & 5.71 & 5.87 \\
\hline $\begin{array}{l}4.92 \\
4.01 \\
2.93 \\
2.70\end{array}$ & & & & & & & & \\
\hline $\begin{array}{r}16.08 \\
4.52 \\
2.50 \\
3.20 \\
2.89 \\
2.76 \\
4.14 \\
2.47\end{array}$ & & & & & & & & \\
\hline 4.34 & 3.60 & 3.25 & 3.36 & 2.86 & 3.28 & 4.38 & 3.31 & 3.72 \\
\hline 3.10 & 1.27 & 1.31 & 1.39 & 1.40 & 1.43 & 1.40 & 1.21 & 1.20 \\
\hline 4.60 & 5.60 & 5.79 & 6.53 & 6.92 & 6.67 & 6.64 & 7.23 & 7.20 \\
\hline 2.34 & 0.53 & 0.78 & 0.94 & 1.14 & 1.16 & 1.19 & 1.01 & 1.29 \\
\hline
\end{tabular}

stages of the economy's growth cycle. At high utilization rates and near the peak of the cycle (when potential output gaps are small), real multipliers will be lower than when capacity is less fully utilized. Timing patterns also are affected; real responses are faster and price increases slower at low rather than high utilization rates.

Aside from the degree of exogeneity of a model and the initial conditions at the time exogenous shifts are introduced, the magnitudes of such changes may influence the sizes of multipliers. With a completely linear model, multipliers depend only on the lag structure and parameters which attach to endogenous and exogenous variables.

For example, in matrix notation, a linear system might take the form:

$$
A+B Y_{t}+\sum_{j=1}^{p} B_{i} Y_{t-j}+C Z_{t}=0
$$

where,

$Y=$ endogenous variables, $y_{i}, i=1 \ldots n$

$Y_{t-j}=$ lagged endogenous variables, $y_{i, t-!}$, with lags $j=1 \ldots p$

$Z=$ exogenous variables, $z_{k}, k=1 \ldots m$

$A, B, B_{j}, C=$ matrices of constant coefficients of orders $1 \times n, n \times n, n \times p$, and $n \times m$, respectively 
TABIE. 1

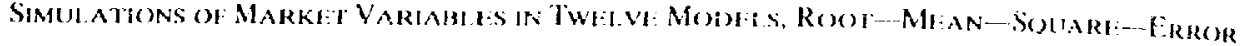

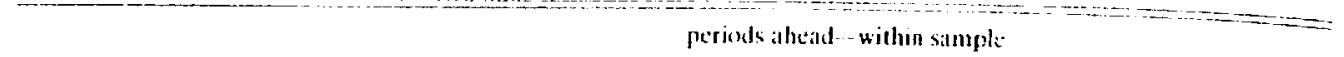

MOPM

DRI

BEA

1

$\begin{array}{llll}p & 0.21 & 0.33 & 0.41\end{array}$

4

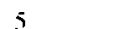

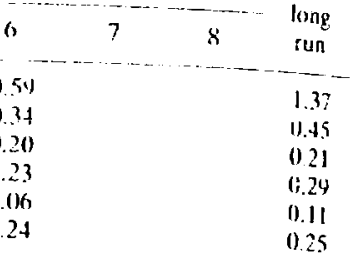

Fair
St. Louis
MPS
Wharton

Wharton

Anticipations

H. C Annuai

\begin{tabular}{|c|c|c|c|c|c|c|c|c|c|}
\hline $\mathbf{P}$ & $(0.20)$ & 0.32 & 0.34 & 0.44 & 0.46 & i). 46 & 0.48 & 0.47 & \\
\hline I!n & 11.25 & 0.34 & 0.18 & $113 \mathrm{~K}$ & 0.39 & (1. 41$\}$ & 0.40 & 0.41 & \\
\hline rs & 0.22 & 0.22 & 0.25 & 0.25 & 0.25 & 120 & (1).26 & 11.20 & \\
\hline IL. & 0.10 & 0.10 & 0.13 & 0.16 & 0.17 & 0.17 & 0.18 & (1.20) & \\
\hline$w$ & 1).(1) & $\{1.11 \mid$ & 0.112 & $11.1)^{2}$ & 0.01 & [i.01] & 0.01 & 0.01 & \\
\hline h & 0.11 & 0.11 & 0.11 & 0.11 & 0.12 & $0.1 ?$ & 0.12 & 0.12 & \\
\hline$P$ & (1. 16 & 10.26 & $0.3 i$ & 0.47 & 0.57 & 0.6 .5 & 0.7 .3 & 0.81 & 1.13 \\
\hline$U_{n}$ & (1). 16 & 1).32 & 0.44 & (J.S) & 0.53 & 0.54 & 11.56 & 0.54 & 0.37 \\
\hline rs & 0.20 & 0.26 & 0.31 & 0.37 & 10.42 & 0.47 & 1).5\% & 0.51 & 0.43 \\
\hline II & (1. 11 & (1. 17 & 11.21 & (1).2.3 & 0.25 & 0.27 & 0.29 & 0.31 & 0.34 \\
\hline$w$ & 0.27 & 0.24 & 0.43 & 0.52 & 0.6 .2 & 0.73 & $0.8 \mathrm{I}$ & 0.89 & 0.43 \\
\hline P' & 0.21 & 1). 34 & 0.44 & $0.5,3$ & 0.57 & 0.57 & 0.54 & 0.57 & 0.77 \\
\hline Un & ().20) & 0.24 & 0.20 & 0.27 & (1.27 & 0.31 & (1. 33 & 0.34 & 0.33 \\
\hline Is & 0139 & 0.50 & 0.52 & 0.57 & 0.61 & 0.61 & 0.58 & 0.57 & 0.59 \\
\hline rL & 0.15 & (1.22 & 0.25 & 0.25 & 0.23 & (1.). I & 0.18 & 0.18 & 025 \\
\hline$w$ & 0.30 & 0.40 & 0.50 & 0.60 & 0.60 & 0.50 & 0.40 & $(0.4 i)$ & 0. $t^{\prime} \mid$ \\
\hline
\end{tabular}

is $\quad 0.19$

0.22

0.24

(1. 32

(1) 55

() 55

(1). 2 !!

0.21

0.20

0.115

0.24

25

\section{Mark III}

C Annua

().21

P 0.19

Un $\quad 0.23$

is 0.34

rI.

$p$

Un 0.21

Un ij.35

is 0.15

rI. $0.1 \mathrm{i}$

$w \quad 1.116$

h 0.11

U

is

rI

w.

P

In $\quad 0.29$

0.21

is $\quad 0.25$

rl.

w

P

Is

rs

rl

h

1.20

0.88

0.45

0. 21

0.04
0.2

0.33

0. 35

0.43

0.31

1..27

0.36

0.17

0.26

0. 44

0.17

0.13

1.23

(1.13

(). 31

(1.39

1). 34

0.22

(I.1) 2

0.31

0.38

(). 33

0. 22

0.02
1.95

1. 15

0.42

0.27

0.06
0.38

0.32

0.34

0.16

0.37

0.48

0.15

(). 13

2.03

0.14

0. .37

0.52

0.36

0.24

0.02

0.36

0.511

0.36

0.24

().112

2.36

1.07

0. 47

(0.33

0.08

11.02
$0.37 \quad 0.37$

0.510 .52

$0.4 .5 \quad 18.55$

$0.35 \quad 0.34$

$0.33 \quad 0.36$

0.160 .16

0.470 .60

$\begin{array}{ll}0.49 & 0.53\end{array}$

0.140 .16

0.150 .14

$2.31 \quad 2.72$

0. 15

$0.49 \quad 0.62$

0.57

0.38

(i) 24

(1.013

0.48

0.56

0.38

0.25

(1).113

2.89

0.42

0.50

0.301

0.09

0.12
0.16

0.24

0.411

0.16

0.64

0.60)

0.16

0.14

2.84

0.17

0.71

0.61

0. 40

0.28

0.03

0.63

0.41

0.31

0.113

0.6(1)

0.61)

0.40

0.28

(1.0)?

0.69

0.63

0.41

0.31

i). 0.3

3.36

3. 34

1. 17

(1.49)

0.44

$\begin{array}{ll}0.11 & 0.12 \\ 002 & 0.02\end{array}$ $\begin{array}{lll}0.82 & 0.98 & 3.10\end{array}$

$\begin{array}{lll}0.17 & 0.19 & 0.33\end{array}$

$\begin{array}{lll}0.44 & 0.50 & 0.55\end{array}$

$\begin{array}{lll}0.19 & 0.22 & 0.41\end{array}$

$0.64 \quad 0.66 \quad 1.61$

$0.67 \quad 0.69 \quad 0.44$

$\begin{array}{lll}0.17 & 0.15 & 0.23\end{array}$

$0.12 \quad 0.10 \quad 0.15$

$2.79 \quad 266 \quad 4.56$

$\begin{array}{lll}0.16 & 0.17 & 0.19\end{array}$

$\begin{array}{lll}0.81 & 0.92 & 1.17\end{array}$

$\begin{array}{lll}0.65 & 0.66 & 0.9 \mathrm{i}\end{array}$

$\begin{array}{lll}0.41 & 0.42 & 0.42\end{array}$

$0.33 \quad 0.35 \quad 0.37$

$0.03 \quad 0.03 \quad 0.02$

$\begin{array}{lll}0.78 & 0.88 & 1.05\end{array}$

$0.6 .5 \quad 0.66 \quad 1.05$

$\begin{array}{lll}0.41 & 0.41 & 0.41\end{array}$

$0.34 \quad 0.36 \quad 0.35$

$\begin{array}{lll}0.0 .3 & 0.03 & 0.02\end{array}$

0.01 


\begin{tabular}{|c|c|c|c|c|c|c|c|c|}
\hline \multicolumn{8}{|c|}{ periods ahead-extrapolation } & \multirow[b]{2}{*}{ Notes } \\
\hline 1 & 2 & 3 & 4 & 5 & 6 & 7 & 8 & \\
\hline 0.25 & $0 . .34$ & 0.57 & 0.98 & 1.65 & 2.44 & & & \multirow{12}{*}{ 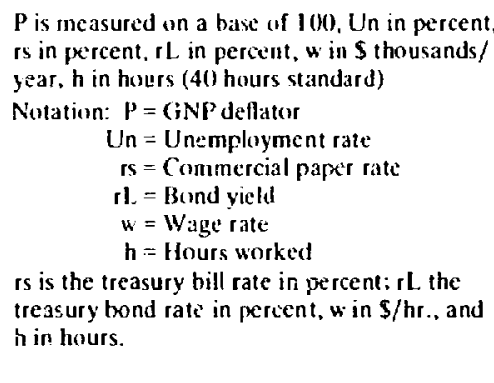 } \\
\hline 0.35 & 0.86 & 1.23 & 1.30 & 1.40 & 1.26 & & & \\
\hline 0.87 & 1.07 & 1.16 & 1.19 & 1.19 & 1.14 & & & \\
\hline 0.42 & 0.52 & 0.60 & 0.68 & 0.75 & 0.79 & & & \\
\hline 0.04 & 0.06 & 0.10 & 0.13 & 0.15 & 0.17 & & & \\
\hline 0.15 & 0.18 & 0.19 & 0.22 & 0.27 & 0.32 & & & \\
\hline 0.42 & 0.65 & 0.80 & 0.91 & 0.96 & 1.08 & 1.30 & 1.70 & \\
\hline 0.26 & 0.51 & 0.81 & 1.02 & 1.16 & 1.26 & 1.14 & 1.21 & \\
\hline 0.43 & 0.48 & 0.50 & 0.52 & 0.54 & 0.60 & 0.70 & 0.77 & \\
\hline 0.28 & 0.40 & 0.45 & 0.51 & 0.54 & 0.61 & 0.64 & 0.76 & \\
\hline 0.02 & 0.02 & 0.03 & 0.04 & 0.05 & 0.07 & 0.08 & 0.10 & \\
\hline 0.17 & 0.22 & 0.23 & 0.30 & 0.3 .3 & 01.33 & 0.31 & 0.311 & \\
\hline 0.39 & 0.61 & 0.75 & 0.86 & & & & & \multirow{5}{*}{$\begin{array}{l}w \text { is an index of rrivate nonfarm } \\
\text { compensation/man hour, 1967:100. }\end{array}$} \\
\hline 0.23 & 0.52 & 0.65 & $(0.79)$ & & & & & \\
\hline 0.54 & 0.53 & 0.59 & 1).66 & & & & & \\
\hline 0.23 & 0.32 & 0.38 & 0.43 & & & & & \\
\hline 0.44 & 0.58 & (j.71 & 0.83 & & & & & \\
\hline 0.21 & 0.39 & 0.57 & 0.76 & 0.97 & & & & \\
\hline 0.36 & 0.68 & 0.90 & 1.08 & 1.23 & & & & \\
\hline 0.48 & 0.81 & 0.90 & 0.76 & 0.71 & 0.70 & & & \\
\hline 0.22 & 0.23 & 0.29 & 036 & 0.34 & 0.30 & & & \\
\hline 1.15 & 1.30 & 1.36 & 1.35 & 1.52 & 1.41 & & & \\
\hline \multirow[t]{2}{*}{0.50} & 0.52 & 0.45 & 0.32 & 0.34 & 0.37 & & & \\
\hline & & & & & & & & $\begin{array}{l}w \text { is measured in cents } \\
h \text { is measured in hours/person/week }\end{array}$ \\
\hline 0.72 & 0.96 & 1.04 & 0.80 & 1.02 & 1.41 & 1.99 & 2.73 & \multirow{10}{*}{$\begin{array}{l}w \text { is measured in } \$ \text { thousands/yr for the } \\
\text { manufacturing sector; } h \text { is measured in } \\
\text { hours ( } 40 \text { hour standard) for the } \\
\text { manufacturing sector. }\end{array}$} \\
\hline 0.52 & 1.11 & 1.63 & 2.00 & 2.42 & 2.83 & 3.10 & 3.29 & \\
\hline 0.77 & 1.10 & 1.33 & 1.51 & 1.57 & 1.70 & 1.86 & 1.93 & \\
\hline 0.23 & 0.35 & 0.39 & 0.49 & 0.56 & 0.64 & 0.75 & 0.89 & \\
\hline 0.07 & 0.09 & 0.11 & 0.11 & 0.16 & 0.22 & 0.27 & 0.32 & \\
\hline $0: 72$ & 0.95 & 1.01 & 0.81 & 1.10 & 1.54 & 2.11 & 2.82 & \\
\hline 0.52 & 1.08 & 1.53 & 1.84 & 2.18 & 2.51 & 2.72 & 2.83 & \\
\hline 0.78 & 1.12 & 1.36 & 1.52 & 1.57 & 169 & 1.84 & 1.90 & \\
\hline 0.23 & 0.35 & 0.40 & 0.50 & (J.57 & 0.64 & 0.75 & 0.88 & \\
\hline \multirow[t]{2}{*}{0.07} & 0.09 & 0.11 & 0.11 & 0.16 & 0.22 & 0.26 & 0.30 & \\
\hline & & & & & & & & $\begin{array}{l}\mathrm{w} \text { is measured in } \$ / \mathrm{hr} \text {. } \\
\mathrm{h} \text { is measured in } 1,000 \mathrm{hrs} / \text { person/yr. }\end{array}$ \\
\hline
\end{tabular}




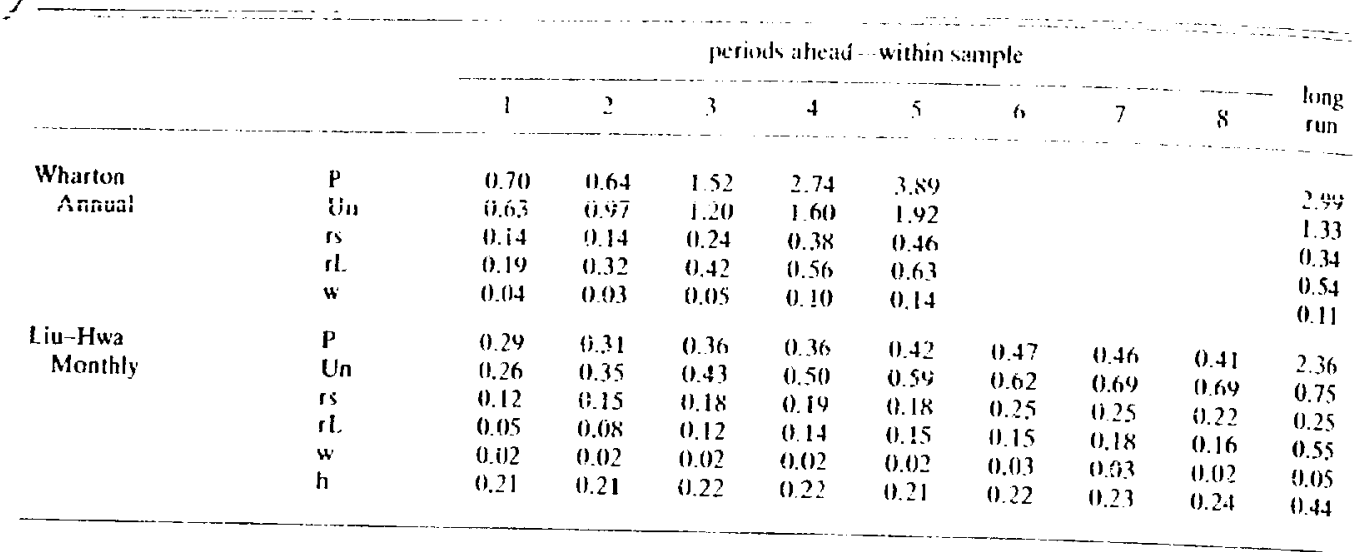

The solution of the system is given by:

$$
Y_{1}=-B^{-1} A-B^{-1} \sum_{j=1}^{p} B_{i} Y_{t-i}-B^{-1} C Z_{t}
$$

Impact multipliers, first period changes in an elenent of the column vector $Y_{\text {, with }}$ respect to changes in an element of $A$ or $Z$, are, respectively,

and

$$
\frac{d y_{i f}}{d a}=b^{i j}
$$

$$
\frac{d y_{i t}}{d z_{k t}}=\sum_{q=1}^{n} b^{i q} C_{q k}
$$

where $b^{i j}$ and $b^{i q}$ are the $(i, j)$ and $(i, q)$ elements of $B^{-1}$. Multi-period impacts of $Z_{t}$ would depend on summed products of elements of $B^{-1}, B_{j}$ and $C$.

However, most econometric models are, to a significant degree, non-linear in variables. For instance, nominal values often are derived by inflating real quantities. Therefore, unless linear approximations are used (which may lead to substantially biased multiplier estimates), numerical methods must be cmployed
to obtain solutions of models and their multipliers.

Finally, the causal nature of models greatly affects multipliers. Reduced from systems generally have vastly different multiplier propertics than models which exhibit more coinplete structural linkages.

The caveats apply to the results shown in Tables 5-8, which report dynamic multipliers over a ten-year interval. Solution periods range from starting near the onset of recessions to the middle of booms. Amounts of exogenous change in non-defense government expenditures vary from $\$ 5$ billion constant (1958) dollars to $\$ 1$ billion in current dollars. Tax and monetary shifts are similarly disparate. Thus, lack of standardization hampers intermodel comparisons.

Despite such differences, however, with the exception of the FRB St. Louis Model, there is a fair amount of agreement among quarterly models. Nominal 


\begin{tabular}{|c|c|c|c|c|c|c|c|c|}
\hline \multicolumn{9}{|c|}{ periods ahead - extrapolation } \\
\hline 1 & 2 & $\therefore$ & 4 & 5 & 6 & 7 & 8 & Noles \\
\hline & & & & & & & & $\begin{array}{l}\mathrm{H} \text { is measured in } \mathrm{S} / \mathrm{hr} \text { in the manufacturing } \\
\text { sector. }\end{array}$ \\
\hline 0.54 & 0.69 & 0.61 & 0.73 & 0.76 & 0.75 & 0.82 & 0.82 & \\
\hline 0.30 & 0.54 & 0.93 & 1.00 & 1.04 & 1.41 & 1.17 & 1.06 & \\
\hline 0.27 & 0.35 & 0.39 & 0.39 & 0.40 & 0.42 & 0.42 & 0.43 & $w$ is measured in $s$ thousands/yr and $h$ in \\
\hline 0.07 & 0.11 & 0.13 & 0.18 & 0.19 & 0.22 & 0.24 & 0.22 & hoies ( 4 ( ) hour standard) \\
\hline 0.03 & 0.0 .3 & 0.04 & 0.04 & 0.03 & (1).04 & 0.04 & 0.04 & \\
\hline 0.20 & 0.20 & 0.15 & 0.19 & 0.12 & 0.13 & 0.12 & (1) 13 & \\
\hline
\end{tabular}

GNP-nondefense government expenditure multipliers are around two after four quarters and then generally continue to rise, with slight fluctuations, thereafter. Results for the annual models and Liu-Hwa monthly model are consistent with this pattern.

Much of the sustained multiplier increase is due to pressures on prices, which appear to accelerate as simulation periods are lengthened. Prices continue to rise despite declines in rates of increase or falls in absolute levels of real output, drops in capacity utilization, and higher unemployment rates. Few of the models contain price anticipation variables and, where they are included, it is doubtful that they are strong enough to account for this phenomenon.

These and related effects are mirrored in the results for constant dollar multipliers ( $\Delta$ real GNP/ $\Delta$ real expenditures). Conventional textbook expositions generally depict real expenditure multipliers approaching positive asymptotes. But, most of the models here show such multipliers reaching a peak in two or three years and then declining (see Table 5). Multipliers for the MPS model decline to negative values quite early, but not as early as the St. Louis Model. At the end of five to ten years, some of the models show that continued sustained fiscal stimulus has ever-increasing perverse effects.

For models in which the stimulus is introduced in nominal terms, the decline in real expenditure multipliers, in part, is attributable to decreasing the amount of real input. That is, the expenditure increase declines in real terms as prices rise. A concomitant effect of the rise in prices is to lower real values of all other exogenous nominal dollar expenditures or transfers. Moreover, in models where government transfers such as current dollar social security payments are endogenous, insufficient allowance probably is made for Congressional actions to raise be nefit levels as inflation erodes reai living standards. Thus, when nominal exogenous stimuli are used in solutions of models, unless upward adjustments in outlays are made for endogenous increases in prices, real stimulus falls and multipliers, as conventionally calculated, will tend to decline after a period of time.

There are also other effects at work. These may be illustrated by the Brookings and Wharton Annual model simulations, wherein government expen- 
TABI.E 5

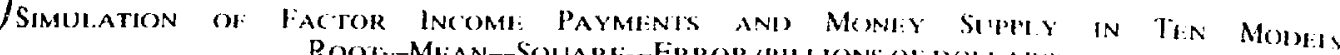
ROOT-MI:AN-SOUARE--FRROR (BHIIIONS OI DOIIARSI

\begin{tabular}{|c|c|c|c|c|c|c|c|c|c|c|}
\hline & & \multicolumn{9}{|c|}{ periods aheald-within sample } \\
\hline & & 1 & 2 & 3 & 4 & 5 & 6 & 7 & $s$ & run \\
\hline $\mathrm{BEA}$ & $\begin{array}{l}\text { PI } \\
W \\
P R \\
M I\end{array}$ & $\begin{array}{l}1.96 \\
1.79 \\
2.44 \\
0.81\end{array}$ & $\begin{array}{l}3.23 \\
2.8 x \\
3.04 \\
1.38\end{array}$ & $\begin{array}{l}4.14 \\
3.55 \\
4.14 \\
1.71\end{array}$ & $\begin{array}{l}+8.10 \\
3.66 \\
+.82 \\
1.8 .3\end{array}$ & $\begin{array}{l}5.52 \\
3.66 \\
5.39 \\
1.94\end{array}$ & $\begin{array}{l}6.31 \\
4.14 \\
5.54 \\
2.15\end{array}$ & & & $\begin{array}{r}11.11 \\
7.77 \\
6.86 \\
3.29\end{array}$ \\
\hline Brookings & $\begin{array}{l}\mathrm{PI} \\
\mathrm{W} \\
\mathrm{PR} \\
\mathrm{MI}\end{array}$ & $\begin{array}{l}1.72 \\
1.79 \\
2.34 \\
0.61\end{array}$ & $\begin{array}{l}2.39 \\
2.63 \\
3.13 \\
1.02\end{array}$ & $\begin{array}{l}2.61 \\
2.95 \\
1.38 \\
1.29\end{array}$ & $\begin{array}{l}2.65 \\
3.09 \\
3.55 \\
1.49\end{array}$ & $\begin{array}{l}2.4 .3 \\
2.95 \\
3.70 \\
1.64\end{array}$ & $\begin{array}{l}2.45 \\
3.03 \\
3.74 \\
1.74\end{array}$ & $\begin{array}{l}2.33 \\
3.60 \\
3.82 \\
1.76\end{array}$ & $\begin{array}{l}2.50 \\
3.05 \\
3.76 \\
1.80\end{array}$ & \\
\hline MOEM & $\begin{array}{l}\text { PI } \\
\text { PR }\end{array}$ & $\begin{array}{l}1.86 \\
1.94\end{array}$ & $\begin{array}{l}3.11 \\
2.34\end{array}$ & $\begin{array}{l}4.85 \\
3.09\end{array}$ & $\begin{array}{l}6.07 \\
3.44\end{array}$ & $\begin{array}{l}6.90 \\
4.00\end{array}$ & $\begin{array}{l}7.56 \\
4.36\end{array}$ & $\begin{array}{l}8.28 \\
4.72\end{array}$ & $\begin{array}{l}8.3 .3 \\
5.06\end{array}$ & $\begin{array}{l}4.57 \\
6.01\end{array}$ \\
\hline DRI & Pl & 4.01 & 4.64 & 5.14 & 5.50 & 5.07 & 4.8 .3 & 4.56 & 4.40 & 4.62 \\
\hline & $\begin{array}{l}\text { PR } \\
\text { MI }\end{array}$ & $\begin{array}{l}2.17 \\
1.12\end{array}$ & $\begin{array}{l}2.55 \\
1.22\end{array}$ & $\begin{array}{l}2.27 \\
1.37\end{array}$ & $\begin{array}{l}3.12 \\
1.41\end{array}$ & $\begin{array}{l}3.44 \\
1.47\end{array}$ & $\begin{array}{l}3.90 \\
1.49\end{array}$ & $\begin{array}{l}4.11 \\
1.57\end{array}$ & $\begin{array}{l}4.24 \\
1.601\end{array}$ & $\begin{array}{l}4.08 \\
1.30\end{array}$ \\
\hline MPS & $\begin{array}{l}P i \\
W \\
P R \\
M I\end{array}$ & $\begin{array}{l}2.00 \\
1.89 \\
2.63 \\
0.62\end{array}$ & $\begin{array}{l}2.66 \\
2.17 \\
3.07 \\
1.00\end{array}$ & $\begin{array}{l}4.30 \\
3.73 \\
3.31 \\
1.34\end{array}$ & $\begin{array}{l}4.73 \\
4.20 \\
3.03 \\
1.53\end{array}$ & $\begin{array}{l}5.72 \\
5.18 \\
3.26 \\
1.63\end{array}$ & $\begin{array}{l}6.34 \\
5.77 \\
3.00 \\
1.66\end{array}$ & $\begin{array}{l}6.62 \\
5.71 \\
3.61 \\
1.68\end{array}$ & $\begin{array}{l}6.51 \\
5.77 \\
2.98 \\
1.69\end{array}$ & $\begin{array}{l}7.94 \\
6.57 \\
3.77 \\
1.64\end{array}$ \\
\hline $\begin{array}{l}\text { Wharton } \\
\text { Mark III }\end{array}$ & $\begin{array}{l}\mathrm{PI} \\
\mathrm{W} \\
\mathrm{PR} \\
\mathrm{MI}\end{array}$ & $\begin{array}{l}1.66 \\
1.37 \\
2.80 \\
0.77\end{array}$ & $\begin{array}{l}2.77 \\
2.56 \\
3.105 \\
1.17\end{array}$ & $\begin{array}{l}3.88 \\
3.66 \\
3.07 \\
1.45\end{array}$ & $\begin{array}{l}4.40 \\
4.17 \\
3.08 \\
1.64\end{array}$ & $\begin{array}{l}4.51 \\
4.30 \\
3.47 \\
1.74\end{array}$ & $\begin{array}{l}4.63 \\
4.37 \\
3.64 \\
1.85\end{array}$ & $\begin{array}{l}4.60 \\
4.21 \\
3.82 \\
1.96\end{array}$ & $\begin{array}{l}4.85 \\
4.31 \\
4.106 \\
2.166\end{array}$ & $\begin{array}{l}6.74 \\
6.92 \\
4.34 \\
2.58\end{array}$ \\
\hline $\begin{array}{l}\text { Wharton } \\
\text { Anticipations }\end{array}$ & $\begin{array}{l}\text { Pl } \\
W \\
P R \\
M I\end{array}$ & $\begin{array}{l}1.59 \\
1.37 \\
2.84 \\
0.78\end{array}$ & $\begin{array}{l}2.61 \\
2.48 \\
2.98 \\
1.18\end{array}$ & $\begin{array}{l}3.65 \\
3.51 \\
2.92 \\
1.44\end{array}$ & $\begin{array}{l}4.14 \\
3.98 \\
2.93 \\
1.62\end{array}$ & $\begin{array}{l}4.21 \\
4.07 \\
3.31 \\
1.74\end{array}$ & $\begin{array}{l}4.30 \\
4.12 \\
3.51 \\
1.87\end{array}$ & $\begin{array}{l}4.23 \\
3.89 \\
3.77 \\
2.91\end{array}$ & $\begin{array}{l}4.45 \\
3.95 \\
3.98 \\
2.11\end{array}$ & $\begin{array}{l}8.39 \\
8.53 \\
4.39 \\
2.29\end{array}$ \\
\hline $\mathrm{H}-\mathrm{C}$ Annual & $\begin{array}{l}\text { Pl } \\
W \\
\text { PR } \\
\text { MI }\end{array}$ & $\begin{array}{l}8.61 \\
7.64 \\
5.114 \\
3.16\end{array}$ & $\begin{array}{l}8.89 \\
7.81 \\
4.45 \\
3.36\end{array}$ & $\begin{array}{l}8.63 \\
6.45 \\
4.78 \\
3.72\end{array}$ & $\begin{array}{r}10.85 \\
8.67 \\
6.49 \\
4.16\end{array}$ & $\begin{array}{r}11.26 \\
9.57 \\
5.29 \\
4.05\end{array}$ & $\begin{array}{r}11.33 \\
9.30 \\
5.35 \\
4.30\end{array}$ & & & $\begin{array}{l}4.83 \\
6.015 \\
6 . \therefore 7 \\
2.44\end{array}$ \\
\hline $\begin{array}{l}\text { Wharton } \\
\text { Annual }\end{array}$ & $\begin{array}{l}\text { Pl } \\
\text { W } \\
\text { PR } \\
\text { MI }\end{array}$ & $\begin{array}{l}2.47 \\
2.46 \\
2.92 \\
2.198\end{array}$ & $\begin{array}{l}4.49 \\
4.74 \\
3.11 \\
2.76\end{array}$ & $\begin{array}{l}9.98 \\
9.93 \\
3.44 \\
2.6 .1\end{array}$ & $\begin{array}{r}14.11 \\
14.13 \\
3.89 \\
2.75\end{array}$ & $\begin{array}{r}21.014 \\
19.67 \\
3.95 \\
3.37\end{array}$ & & & & $\begin{array}{r}18.86 \\
17.91 \\
3.37 \\
1.23\end{array}$ \\
\hline $\begin{array}{l}\text { Liu-Hwa } \\
\text { Monthly }\end{array}$ & $\begin{array}{l}P 1 \\
w \\
P R \\
M !\end{array}$ & $\begin{array}{l}3.86 \\
2.02 \\
1.62 \\
0.74\end{array}$ & $\begin{array}{l}4.11 \\
2.18 \\
2.53 \\
0.91\end{array}$ & $\begin{array}{l}4.31 \\
2.42 \\
2.91 \\
0.97\end{array}$ & $\begin{array}{l}4.32 \\
2.65 \\
3.4 ! \\
1.28\end{array}$ & $\begin{array}{l}4.58 \\
2.61 \\
3.21 \\
1.08\end{array}$ & $\begin{array}{l}4.53 \\
2.75 \\
.3 .58 \\
1.54\end{array}$ & $\begin{array}{l}4.27 \\
2.91 \\
3.72 \\
1.48\end{array}$ & $\begin{array}{l}5.24 \\
2.97 \\
3.94 \\
1.84\end{array}$ & $\begin{array}{l}7.41 \\
8.08 \\
5.72 \\
2.94\end{array}$ \\
\hline
\end{tabular}

diture inputs are stated in real terms. Here, too, real expenditure multipliers rise to a peak and then begin to fall. While there probably are some multiplier feedbacks on some exogenous expenditures and transfers from prices, the primary cause of the fall-offs in multipliers after two years in these models most likely is due to capacity constraints and reductions in rates of increase of business fixed and inventory investment. Only in the Fair model does the real multiplier fail to drop. This model has only a short solution horizon and some nonresponsive anticipatory variables. Although the real multiplier drops in the $\mathrm{H}-\mathrm{C}$ Annual model, this
becomes apparent only after 14 years. 


\begin{tabular}{|c|c|c|c|c|c|c|c|c|}
\hline \multicolumn{8}{|c|}{ periods ahead-extrapolation } & \multirow[b]{2}{*}{ Notes } \\
\hline 1 & 2 & 3 & 4 & $\xi$ & 6 & 7 & 8 & \\
\hline 2.68 & 5.44 & 15.73 & 14.33 & 2181 & 22.93 & & & \multirow{4}{*}{ Notation: $\begin{aligned} \mathbf{P 1} & =\text { Personal lncome } \\
\mathbf{W} & =\text { Eniployee compensation } \\
\mathbf{P R} & =\text { Corporate Profits before tax } \\
\mathbf{M I} & =\text { Currency and demand depisils }\end{aligned}$} \\
\hline 4.00 & 12.32 & 19.59 & 23.60 & 25.90 & 26.65 & & & \\
\hline 3.17 & 5.48 & 4.55 & 4.17 & 3.57 & 4.46 & & & \\
\hline 2.36 & 4.59 & 6.66 & 8.24 & 9.47 & 10.44 & & & \\
\hline 3.43 & 6.20 & 9.6 .3 & 12.67 & 16.04 & 19.15 & 21.50 & 25.38 & \multirow{18}{*}{$\begin{array}{l}\text { Demand dequsits withoul the atidition of } \\
\text { currency are used for Ml. Currency is } \\
\text { separaitly tabulated. }\end{array}$} \\
\hline 3.22 & 6.24 & 9.63 & 12.91 & 15.86 & 18.97 & 20.88 & 24.36 & \\
\hline 3.82 & 5.70 & 7.82 & 10.26 & 12.68 & 14.17 & 15.81 & $17.5 i 1$ & \\
\hline 1.23 & 2.49 & 3.80 & 5.31 & 7.13 & 9.28 & 11.59 & 14.16 & \\
\hline 4.87 & 7.22 & 7.86 & 10.92 & & & & & \\
\hline 4.06 & 5.20 & 5.24 & 5.22 & & & & & \\
\hline 13.13 & 22.30 & 29.54 & 33.24 & 41.49 & 49.16 & 55.61 & 61.56 & \\
\hline 10.08 & 18.39 & 25.14 & 28.61 & 36.41 & 43.84 & $5(0.92$ & 55.91 & \\
\hline 6.91 & 9.28 & 9.00 & 7.95 & 7.63 & 6.82 & 6.72 & 7.16 & \\
\hline 2.32 & 2.01 & 2.28 & 3.28 & 4.87 & 5.86 & 7.20 & 8.64 & \\
\hline 13.40 & 22.14 & 28.60 & 31.57 & 38.93 & 45.76 & $5 ! .16$ & 55.82 & \\
\hline 10.12 & 18.09 & 24.21 & 26.96 & $34 .(k)$ & 40.55 & 45.67 & 50.27 & \\
\hline 7.39 & 9.53 & 8.25 & 6.77 & 6.26 & 5.46 & 6.13 & 7.88 & \\
\hline 2.52 & 2.21 & 2.14 & 2.97 & 4.50 & 5.32 & 6.41 & 7.67 & \\
\hline 7.83 & 8.75 & 11.96 & 10.00 & 7.24 & 14.67 & 9.61 & i) .22 & \\
\hline 3.77 & 3.79 & 4.46 & 3.61 & 3.63 & 3.56 & 3.21 & 3.54 & \\
\hline 5.84 & 5.54 & 4.25 & 5.44 & 5.80 & 4.29 & 5.49 & 5.07 & \\
\hline 2.21 & 1.90 & 2.83 & 1.92 & 2.71 & 2.13 & 2.33 & 2.55 & \\
\hline
\end{tabular}

Economic theory also suggests that declines in real multipliers could be caused by financial stringency if monetary authorities do not curtail rising interes rates by expanding bank reserves so as to support ever higher financial transactions and investment demands. As can be seen by comparing results shown in Tables 6 and 7, an accommodating monetary policy of constant interest rates tends to raise long-term expenditure multipliers but does not alter the basic pattern of movement to a peak and then decline.

Multipliers for decreases in personal taxes are shown in Table 8 . In the first few years, nominal GNP-tax multipliers rise more slowly than nominal GNP- 
expenditure multipliers, but surpass the latter in the BFA and MOFM models atter seven ycars. Reat (iNP-lax multipliers in all the models joak attel iwo to three years, but are signiticantly lower (by 0.3 to 0.9 ) than real GNP-cxpenditure multiplicrs. This is not unexpected. The differences betwecn expenditure and tax multipliers need not necessarily equal unity. They do so only in simplistic balanced-budget models that exclude a multiplicity of leakages and incomeexpenditure feedbacks. (For a proof, see G. Fromm and P. Taubman; for examples of policy simulations with balanced budget strategies, sec V. 1)uggal.) ${ }^{3}$

Aside from first-round effects in multiplier calculations, government expenditure changes (of a constant avcrage mix) probably are more powcrful than persona! income tax changes over a period of a few ycars because shifts in government outlays tend to be more intensive in gencrating privatc investment than comparable amounts of personal income tax increase or reduction. This advantage persists in the Brookings. DRI. Wharton Anticipation, H-C Annual, and Wharton Annual models but disappears in the BEA, MQEM, MPS, and Wharton Standard models. In fact, real GNP-real tax multipliers are higher (for some models, less negative) for the latter models af ter from three to seven years. This occurs because non-linear impacts of capacity constraints and price effects are different in these than in the former models.

There are even more strifing disparities between models in inultiplier responses to shifts in monetary policy. With the exception of the FRB St. Louis Model (in which demand deposits and currency are augmented), an exogenous increase of either $\$ 0.5$ billion or $\$ 1.0$ billion in unborrowed reserves is introduced in each model. As can be seen in Table 9, this has virtually no short-run or long-run effect on nominal GNP in the BEA moilel and an ever-increasing (at least over five years) in the MPS and Wharton Annual models. ${ }^{4}$ In the DRI. Wharton standard, and H-C Annual models, the nominal GNP-nominal money multiplier peaks after two to three years and then begins to decline in a cycling
path.

Real GNP-nominal money multipliers reflect thesc same patterns over the first few years, but because of rises in prices, multipliers are lower thereafter. Prices apparently rise fastes! in the DRI and FRB St. Louis models, since real GNP-nominal money multipliers become negative after four or five years.

\section{Prospects}

This summary report marks the end of a second phase of comprehensive analysis of American econometric models. In the first phase a number of U.S. ${ }^{3}$ G. Fromm and P. Taubman. Policy Simulaions with an Econometric Model (Amsterdam:
North-Holland. 1967).

V. Duggal. "Fiscal Policy and Economic Stabilization." The Brookings Model-Perspectite and Recent Developments. eds. G. Fromm and l.. R. Klein (.Anstcrdam: North-1 Holland, 1975). significantly stronger.

$s$ it is encourat. attractive to model builders in that the format of our research discussions and project planning are conference: Canadian model builders have attended apanese model proprictors have held a similar builders have considered holding similar comparative mectings as guests: and European model seminars have felt that much was gained in the information exchanges in these nodel comparisen
seminars. 
models were examined in detail for cyclical content, (1969) followed soon after by a sinular examination of price determination, 1970. "These two investigationsboth conferences-looked carefully into cross-model comparisons for specific characteristics. The Seminar on Model Comparisons in a series of papers in Internaiional Economic Review (June, 1974, October, 1974, February, 1975) and in the present paper looked at a wider variety of model properties in a crosssection analysis. What remains for future rescarch in this area?

New topics for discussion have enlarged our agenda as follows:

Turning point analysis
Ex ante error analysis
Error decomposition
Comparative policy simulation
Added information through model combination

Models perform less well in the neighborhood of critical turning points than along sustained monotonic paths of expansion or decline. Much is to be learned about model performance in seeing whether direction and magnitude of change at peaks and troughs is correctly simulated. A step in turning point research has already been taken by Adams and Duggal and reported in their analysis of the Wharton Model (anticipation version) contained in the IER symposiumı. ${ }^{7}$ There was prior consideration of this matter in the 1969 Conference. Now that the U.S. economy is in the midst of a major recession, we are having an unusual opportunity to examine extreme turning points in great detail. When the cycle has completed its course, it will be a good time to look back and see what has been learned about turning point performance.

The Seminar has concentrated attention primarily on sample period and expost extrapolation error. A number of individual model proprietors have been making their own examinations of ex alite forecast error. Additionally, some outsiders have tried to make independent assessments of forecast error. As these parties often lack the familiarity with the models that only the proprietors can acquire in daily use, some of these error calculations encounter the pitfalls pointed out in a general paper at the beginning of the symposium. ${ }^{8}$ Accordingly, the participants in the Seminar on Model Comparisons are designing an internal study for the analysis of ex ante forecast errors.

Errors are studied only partly for their own sake; they are most useful as a guide to model improvement by showing where deficiencies occur. To be most helpful in this respect, errors should be decomposed into the parts due to (1) coefficient uncertainty, (2) residual disturbances, (3) errors in forecast input values (initial conditions and exogeneous variables) (4) misspecifications of the

\footnotetext{
${ }^{6}$ Economic Models of Cyclical behamior, ed. B. G. Hickman (N.Y., National Bureau of Economic Research, 1972); Econometrics of Price Determination, ed. Otto Eckstin (Washington, D.C.: Federal Reserve Board, 1972).

${ }^{7}$ F. G. Adaums and Vijaya Duggal, "Anticipations Variables in an Econometric Model: Performance of the Anticipations Version of Wharton Mark III," International Economic Review, 15 (June, 1974).

${ }^{*}$ E. P. Howrey, L. R. Klein, and M. D. McCarthy, "Notes on Testing the Predictive Performance of Econometric Models," International Economic Review, 15 (June, 1974), 366-83.
} 


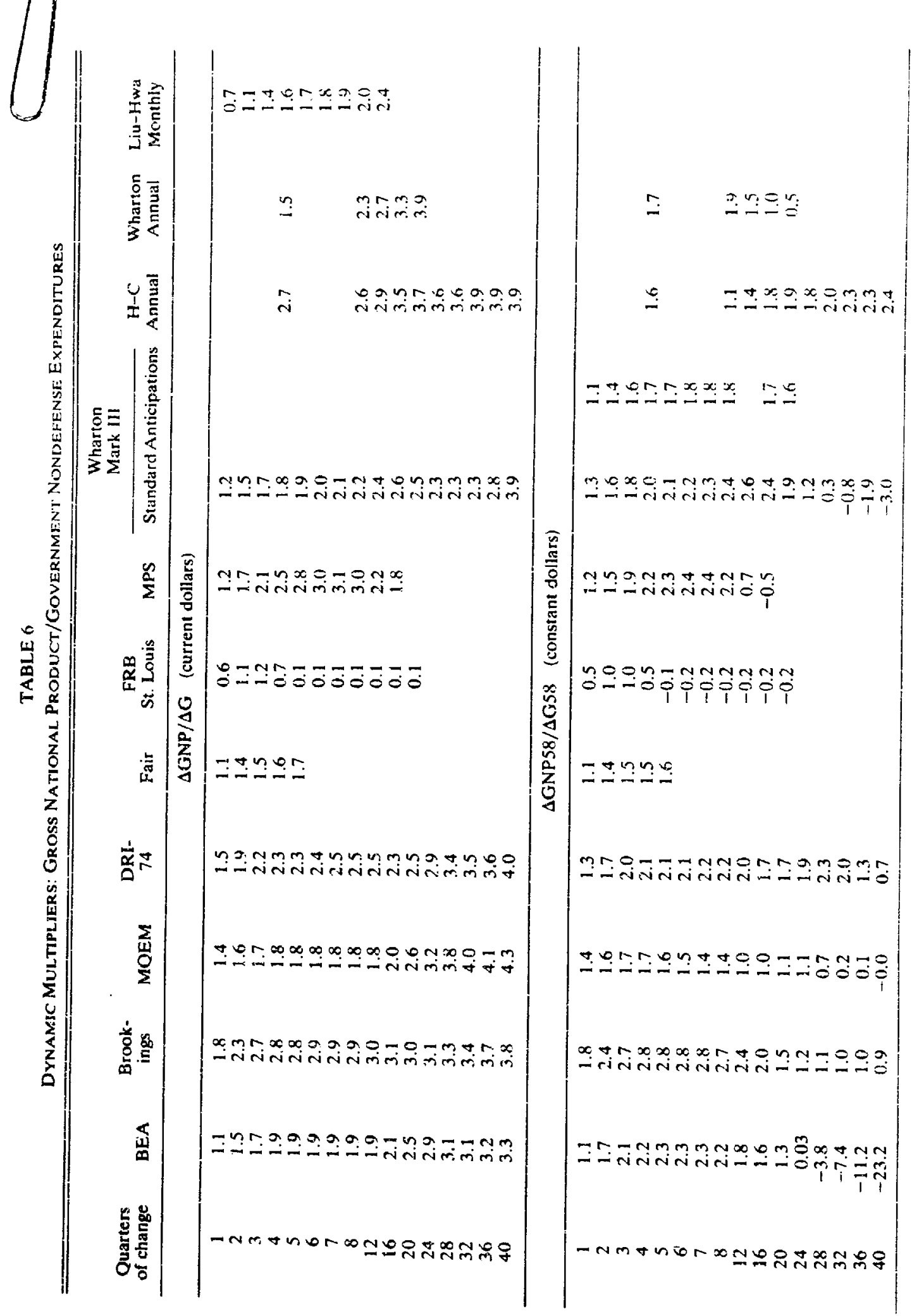




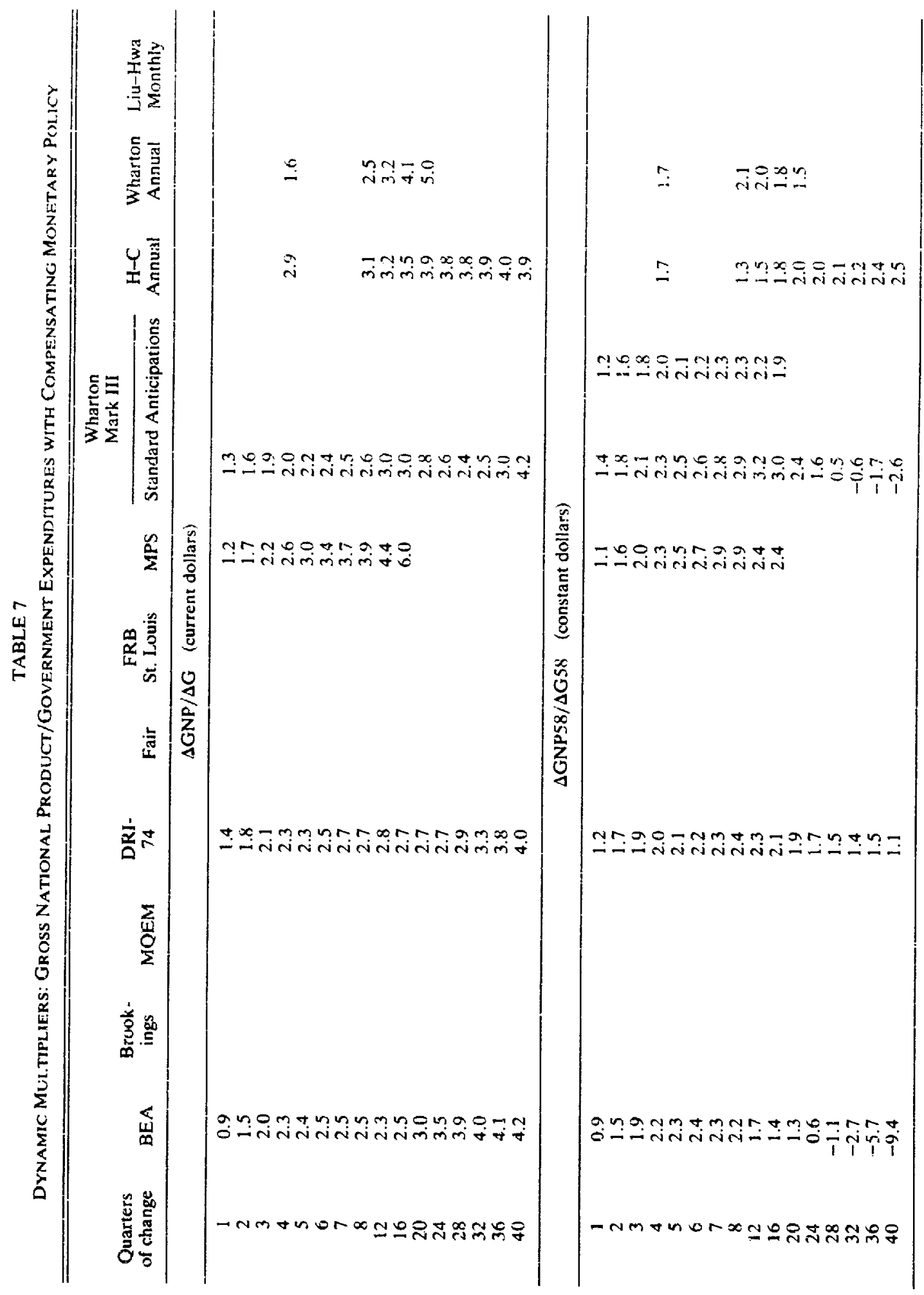




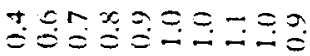

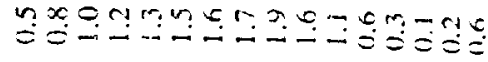

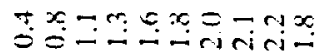

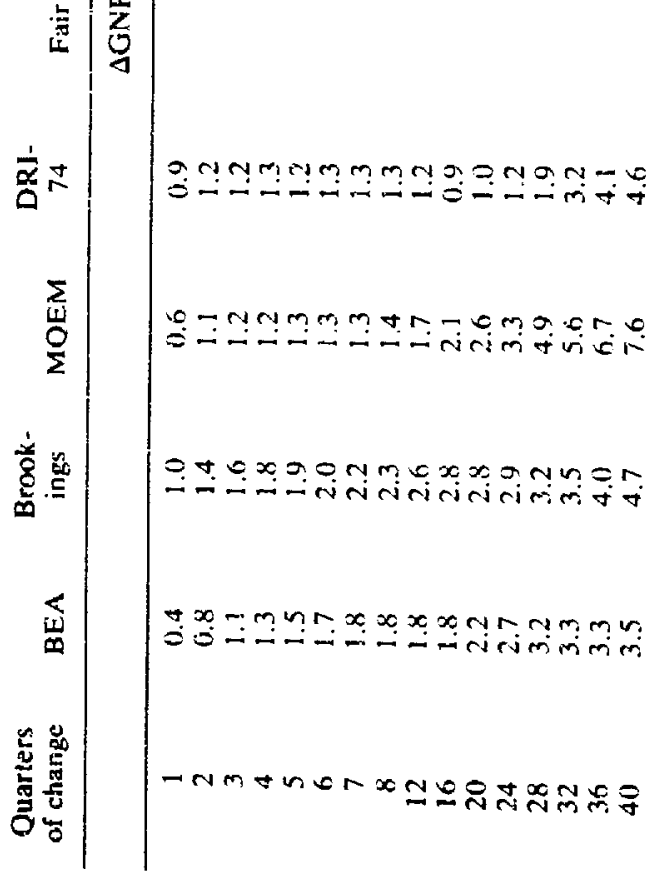

อำำำ

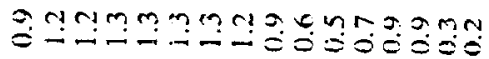

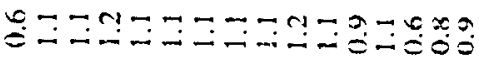

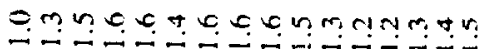

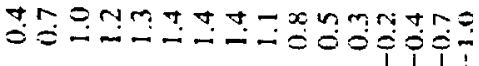




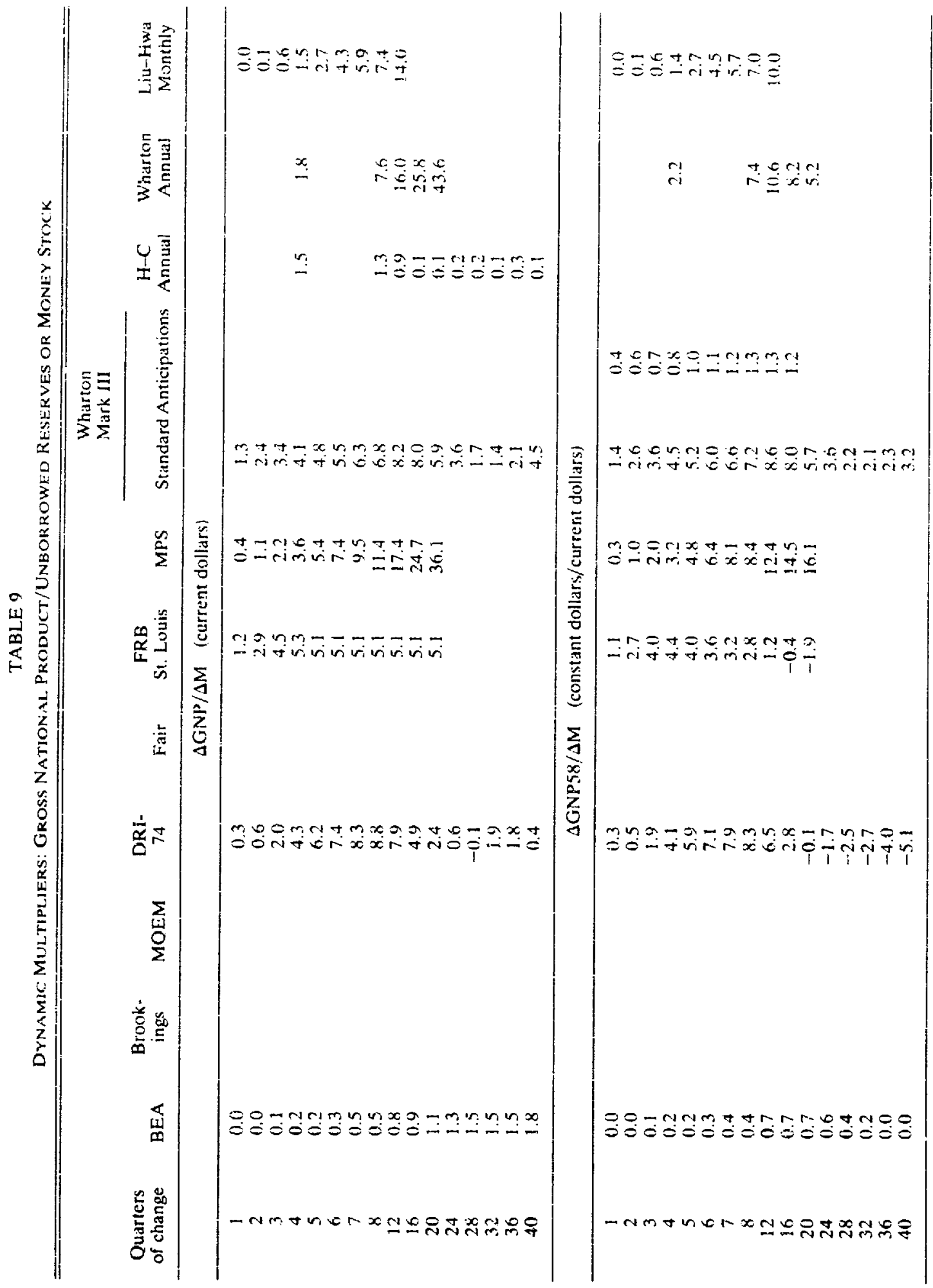


BEA Model: Period 1962-71. Increase of $\$ 1$ billion in federal nondefense expenditures; proportion due to compensation of government employees based on 1962-71 actual data. $\$ 1$ billic:n (1958 dollars) decrease in personal taxes. Increase of $\$ 0.5$ billion in unborrowed reserves.

Brookings Model: Pcriod 1956:1-1965:4. Increasc of $\$ 5$ billion (1958 dollars) in governnent expendiures; decrease of $\$ 5$ billion in personal taxes. Tà inultiplic r computed as ratio lu denaled and undeflated values of $\$ 5.0$ billion, respectively.

MQEM Model: Period 1962:1-1971:4. \$1 billion increase in nondefense expendilures; decrease of $\$ 1$ billion in personal taxes.

DRI-74 Model: Period 1961:1-1970:4. Increase of $\$ 5$ billion (1958 dollars) in federal nondefense expenditures. Decrease of $\$ 5$ billion in personal taxes. Increase of $\$ 1.0$ billion in unborrowed reserves.

Fair Model: Period 1962:1-1963:1. \$1 billion increase in nondefense expenditures: anticipations variables are exogenous. No tax variables in model.

FRB St. Louis Model: Period 1962:1-1966:4. \$5 billion increase in nondefense expenditures. Inctease of $\$$ (t. 5 billion in $\mathrm{Ml}$.

MPS Model: $\$ 1$ billion increase in exports without accommodating monetary policy and \$1 billion decrease in personal taxes. Increase of $\$ 0.5$ billion in unbror rowed reserves.

Whar ton Mark III Model: Period 1965:1-1974:4. Incre ase of $\$ 1$ billion in nondefensc expenditures with average associated change in government wage bill and employment; decrease of $\$ 1$ billion in personal taxes. Increase of $\$ 0.5$ billion in unborrowed reserves.

H-C Annual Model: Period 1951 -66. Increase of $\$ 1$ billion in nondefense expenditures. Interest rates are endogenous. Decrease of $\$ 1$ billion in personal taxes. Intrease of $\$ 0.5$ billion in unborrowed reserves.

Wharton Annual Model: Period 1962-66. Increase of $\$ 5$ billion (1958 dollars) in nendefense expenditures with average associated change in government wage bill and employment; decrease of $\$ 1$ billion in personal taxes. Increase of $\mathbf{\$ 0 . 5}$ billion in unborrowed reserves.

Liu-Hwa Model: Period 1961:01-1964:06. Increase of $\$ 1$ billion in nondefense spending. Decrease of $\$ 1$ billion in personal taxes. Increase of $\$ 1$ billion in unborrowed reserves.

equation system. The analysis of error is being designed so as to bring these different sources into display for separate measurement.

Although we have not achieved as much model uniformity as we wanted for the calculations discussed in this summary paper, we have come far in this direction. Cross-model comparison has been done only for multiplier and historical error analysis, but the Seminar is now embarking on a new investigation of alternative policy analysis, particularly for the historical phase, 1965-75. Comparabie changes in monetary, fiscal, and trade policies, as compared with those actually followed in this period, are being introduced into the several models to see if there is any consensus as to what public authorities might have or should have done to have avoided or mitigated the inflation-recession condition in which we now find ourselves (1974-75). These will be presented in another Seminar symposium. ${ }^{\circ}$

The different models in this large Seminar collection are all viewing the working of the economy through sonewhat different mechanisms--different approximations to reality. Each has some special characteristics, and each has some unusual insight. A combination of model results may prove to be more effective than any one set in interpreting movements in the economy. A study to seek improved or "optimal" combinations of model results is presently being
initiated.

\footnotetext{
${ }^{9}$ Results are to be reported at the December 1975 meetings of the American Economic Association and a summary is to appear in the May, 1976 American Economic Review.
} 
These are only some of the findings and lines of research that could be pursued by this unusual Seminar of model builders. As ever, there is much to be done, much more scope for standardization, and much room for improvementboth in model structure and results.

\author{
National Bureau of Economic Research \\ University of Pennsylvania
}

\title{
Bibliograpiy
}

Adams, F. Gerard and Vijaya G. Duggal, "Anticipations Variables in an Econometric Model: The Anticipations Version of Wharton Mark III," International Economic Review, XV (June, 1974), 267-284.

Andersen, L. C. and K. M. Carison, "A Monetarist Model for Economic Stabilization," Federal Reserve Bank of St. Louis Review, LII (April, 1970), 7-25.

-_. "St. Louis Model Rcvisited," International Economic Review, XV (June, 1974), 305-327.

Ando, Albert, "Equations in the MIT-PENN-SSRC Econometric Model of the United States," (mimeographed), Univcrsity of Pennsylvania (January, 1973).

___ "Some Aspects of Stabilization Policies, the Monetarist Controversy, and the MPS Modcl," International Economic Review, XV (October, 1974), 541-571.

- and F. Modigliani, The MPS Econometric Model: Its Theoretica! Foundation and Empirical Findings, forthcoming in 1975.

Coen, Robert M., "Labor Force and Unemployment in the 1920's and 1930's: A Re-Examination Based on Postwar Experience," The Review of Economics and Statistics, LV (February, 1973), 46-55.

De Leeuw, Frank and Edward Gramlich, "Thc Federal Reserve--MIT Econometric Model," Federal Reserve Bulletin, LIV (Washington, D.C.: Board of Governors of the Federal Reserve System, January, 1968), $1 \mathrm{i}-40$.

Dhrymes, Phoebus J., et. al., "Criteria for Evaluation of Econometric Models," Annals of Economic and Social Measurement (July, 1972), 291-324.

Duesenberry, J. S., G. Fromm, L. R. Klein, and E. Kuh, eds., The Brookings Quarterly Econometric Forecasting Model of the United States (Chicago: Rand McNally and Co., 1965).

Duggal, V., "Fisca! Policy and Economic Stabilization," 'The Brookings Model: Perspective and Recent Developments, eds., G. Fromm and L. R. Klein (Ansterdam: North-Holland, 1975).

_-, Iawrence R. Klein, and Michael D. McCarthy, "The Wharton Model Mark III: A Modern IS-LM Construet," International Economic Review, XV (October, 1974), 572-594.

Eckstein, Otto, ed. Econometrics of Price Determination, (Washington, D.C.: Federal Reserve Board, 1972).

-_. Edward W. Green, and Allen Sinai, "The Data Resources Model: Uses, Structure and Analysis of the U.S. Economy," International Economic Review, XV (October, 1974), 595-615.

Evans, Michael K. and Lawrence R. Klein. The Wharton Econometric Forecasting Model (Philadelphia: Fconomics Research Unit, Wharton School of Finance and Commercc, University of Pennsylvania, 1968).

Fair, Ray C., A Short-Run Forecasting Model of the United States Economy (Lexington: D.C. Heath and $C o ., 1970$ ).

- "An Evaluation of a Short-Run Forecasting Model," International Economic Review, XV (June, 1974), 285-304.

Fromm, G. and P. Taubman, Policy Simulations with an Econometric Model (Amsterdam: NorthHolland, 1967).

_. Lawrence R. Klein, and George R. Schink, "Short- and Long-Term Simulations with the Brookings Model," in Bert G. Hickman, ed., Econometric Models of Cyclical Behavior, Volume I, Studies in Income and Wealth (New York: National Bureau of Economic Research, 1972).

Fromm, G., "Implications to and from Economic Theory in Models of Complex Systems," American Journal of Agricuitural Economics, May 1973, pp. 259.71.

Hickman, Bert, ed., Economic Models of Cyclical Behavior (N.Y., National Bureau of Economic Research, 1972). 
Michael D. Hurd, and Robert M. Coch. "The Itictiman-iocn Annual Cirowill Motel: Stuctural Characteristics and Policy Responses," Internetiemal ficonmic Rebiew. XVI (Fef)mairy, 1975).

Hirsch, Albert A., M. Lichenberg. and (;. R. Green, "The BI A Ouarterly Ecomometric Model,"

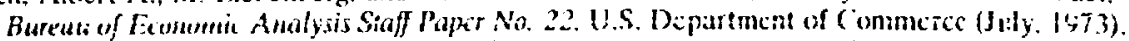

Bruce T. Grimm, and Gorti V. L. Nar asimhan, "Some Multiplier and Error Characteristics of the BE:A Quarterly Model." International Ficmomic Review. XV (October, 1974). 616-631.

Howrey. F. P., I., R. Klein. and M. D. Mc arthy. "Nostes on lesting the Predictive Performance of Econometric Models," International tconomic Review. 15 (Junc. 1974), 366-8,3.

Hymans. Saul H., and Harold T. Shapiro. "The DHL. III Ouarterly I:conometric Model of the U.S. Economy." Research Seminar in Ouantitative Economies, The University of Michigan, Ann Arbor. (197!).

. "The Structure and Properties of the Michigan Quarterly Lconometric Modei of the U.S. Economy." International Economic Review. XV (Octuber, 1974), 632-653.

Liebenberg. M. A. A. Hirsch, and J. Popkin. "A Quarterly Econonetric Model of the United States: A Progress Report." Survey of Curreni Business. XLVI (May. 1966). 13-39.

Litr. Ta-Chung and Erh-Cheng Hiwa. "Structure and Applications of a Monthly Econometric Model of the U.S. Fionomy." International Eionamic Review. XV (Jume, 1974), 328-36.5. - "A Monthly Econometric Model of the U.S. Economy."

Mcearthy. Michaef D.. "The Wharton Mark III Econonetric Mextel of the United States," Ecconomic Research Unit. University of Pennsylvanial. 1972.

Preston. R. S.. "The Whartol Annual and Industry Forecasting Model." liconomics Rescarch Unit. Departinent of Fconomics. Liniversity of Pennsylvaniat. (1972).

received May 1975

revised October 197.5 